\title{
The Power of Whispers:
}

\section{A Theory of Rumor, Communication and Revolution*}

\author{
HENG CHEN \\ University of Hong Kong \\ YANG K. LU \\ Hong Kong University of Science and Technology \\ WING SUEN \\ University of Hong Kong
}

August 28, 2014

\begin{abstract}
We study how rumors mobilize individuals who take collective action. Rumors may or may not be informative, but they create public topics on which people can exchange their views. Individuals with diverse private information rationally evaluate the informativeness of rumors about regime strength. A rumor against the regime can coordinate a larger mass of attackers if individuals can discuss its veracity than if they cannot. Communication can be so effective that a rumor can have an even greater impact on mobilization than when the same story is fully believed by everybody. However, an extreme rumor can backfire and discourage mobilization.
\end{abstract}

Keywords. global game, public signals, swing population, mixture distribution, censorship

JEL Classification. D74, D83

${ }^{*}$ We thank Roland Bénabou, Matthias Doepke, Christian Hellwig, Satoru Takahashi, Jianrong Tian, Yikai Wang, Fabrizio Zilibotti, and seminar participants at NBER Summer Institute 2012, UBC-HKU Microeconomics Workshop, Society for Economic Dynamics 2013 Meeting, University of Hong Kong, Toulouse School of Economics, European University Institute and National University of Singapore for their suggestions. Guangyu Pei deserves praise for providing excellent research assistance to this project. Our research is partially funded by the Research Grants Council of Hong Kong (Project No. HKU 742112B). 


\section{Introduction}

Collective actions, such as riots, currency attacks and bank runs, are often immersed in rumors. Perhaps the most dramatic place to witness rumors in action is a political revolution. Amid the recent Tunisian revolution, Ben Ali, the ex-Tunisian leader, was said to have fled his country. This was confirmed after conflicting rumors about his whereabouts, and finally led to the end of street protests. A while later in Egypt, it was widely reported that Mubarak's family had left for London, which was believed by many as a clear sign of fragility of the regime. Similar rumors about Qaddafi and his family appeared in Libya when the battle between the opposition and the regime intensified. Rumors are not limited to the series of revolutions in the Arab Spring. During the 1989 democracy movement in China, rumors repeatedly surfaced about the death of the leaders, Deng Xiaoping and Li Peng, as well as the divide among communist leaders. ${ }^{1}$

Are rumors just rumors? In many cases, yes. Rumors that spread during turmoils often disappear quickly without a trace. This seems to be natural, as rational individuals may discount unreliable information they receive in those situations. However many historical incidents suggest that rumors often turn out to be particularly effective in mobilization. The Velvet Revolution in Czechoslovakia was described as a "revolution with roots in a rumor" (Bilefsky 2009). At the dawn of the revolution, a prominent (false) rumor that a 19-year old student was brutally killed by the police triggered many otherwise hesitant citizens to take to the streets. The revolution gained huge momentum right after that and the regime collapsed a few days later. In the Arab Spring, the news about Mubarak's family proved to be false, yet the opposition credited it for "mark[ing] a new phase" in their campaign. ${ }^{2}$ Chinese history also offers many anecdotes in which rumors mobilized mass participation, including the Boxer Uprising, the Republican Revolution, and the May Fourth Movement (Zhang 2009). Similarly, riots are often amplified or even sparked by rumors as well: the 1921 Tulsa race riot, the 1967 Newark riot and the 2004 Rome riot provide dramatic examples.

A common interpretation of the role of rumors in mass movements is that individuals are just blindly herded by them. However we adopt the position that individuals are fully aware that rumors circulating in times of turmoil may or may not be well founded, and that they update their beliefs in a Bayesian manner. Since rumors are

\footnotetext{
${ }^{1}$ There were widespread rumors of many variants that Deng died of illness during the protest and that $\mathrm{Li}$ was shot to death. It was also widely rumored in the media that some senior leaders in the Communist Party wrote an open letter to oppose taking any action against students. See, for example, the news story in the daily newspaper Ming Pao on June 6, 1989.

${ }^{2}$ World Tribune reported on January 28, 2011 that "confirmed by a Western diplomat, ... Mubarak's wife, Suzanne, son, Gamal, and granddaughter arrived in London on a private jet as Egypt's defense minister secretly flew to the United States."
} 
widely circulated and commonly observed, they may serve as a coordination device just like a public signal in a coordination game. We explain why some rumors are effective in mobilizing participation in collective actions while others are not.

In this paper, we focus on two key aspects of rumors: that they may be true or false, and that people talk about them. Individuals in times of uncertainty and crisis often seek others' opinions and discuss with peers about their judgment and evaluation of rumors. Information from fellow citizens can influence their beliefs and even actions. The core of our paper is to show that communication among individuals centering around a public topic can substantially change outcomes of collective actions.

Specifically, we model political revolution as a global game. Citizens are uncertain about the regime's strength and possess dispersed private information about it. A citizen's incentive to revolt increases with the aggregate action of all other citizens. If there are sufficient participants, the regime collapses; otherwise it survives. Before citizens take actions, they hear a rumor about the regime. This rumor is a publicly observed message, which could be either an informative signal about the regime's strength or an uninformative noise unrelated to fundamentals. Citizens assess the informativeness of the rumor based on their private information. As a consequence of diverse private information among citizens, their assessments may also differ. Further, citizens communicate with one another and tell their peers whether they believe the rumor or not.

In this model, the degree of skepticism is endogenous: citizens whose private information differs more from the rumor are more skeptical of it. Due to this skepticism, rumors against the regime mobilize fewer attackers than when such news is known to be trustworthy. If a rumor is far different from the fundamental, it will also differ from most citizens' private information and therefore be heavily discounted by them. As a result, extreme rumors have little impact on equilibrium outcomes.

When citizens communicate, those whose private information is close to the rumor will tell their peers that the rumor is informative. Recipients of confirmatory messages treat what their peers say as evidence for the truth being close to what the rumor suggests, and therefore become more responsive to the rumor. Consider, for example, a rumor against the regime. A fraction of the population (those with intermediate private information) will attack the regime if their peers tell them that the rumor is informative, and will not attack otherwise. If the rumor is indeed near the true strength, more citizens will receive confirmatory messages from their peers. Therefore, communication helps such a rumor to mobilize more attackers. By the same mechanism, if the rumor is far from the truth, most citizens will express disbelief to their peers, which discourages attacking. Therefore, communication overcomes or reinforces skepticism 
about a rumor, depending on whether the rumor is close to the fundamental or not.

Interestingly and surprisingly, we find that communication could make rumors even more powerful than trustworthy news in mobilizing individuals. For the same news against a regime, it is possible that the regime would survive if all citizens believe that the news is informative and fully trustworthy, but would collapse if citizens are skeptical about its veracity and talk about it. In the latter scenario, recipients of confirmatory messages react to the rumor very strongly, and the mass of those recipients is considerably large.

Our work enriches the global games literature (Morris and Shin 2003) in a couple of directions. We offer a specification of public signals using mixture distribution that allows us to capture people's skepticism. Specifically, by allowing an additional layer of Bayesian updating on the "quality" of the information source, our model captures the fact that individuals tend to discount information which differs too much from their priors. ${ }^{3}$ We also show that this specification is qualitatively different from having a public signal with low precision. ${ }^{4}$ In our model, the dispersion in private information is crucial, not only because it guarantees equilibrium uniqueness, but also because it generates diverse assessments on the informativeness of rumors, which provides a ground for the study of communication among citizens. It is the core of this paper.

In much of the global games literature, citizens are assumed to only respond to the signals they observe; any further interactions among citizens are often left out for simplicity. In reality, individuals do exchange information with one another before they make decisions and take actions. This is especially true in collective actions such as protests, demonstrations and revolutions. We model direct interaction between citizens by allowing them to communicate privately, rather than just observing a public signal of what others are doing. ${ }^{5}$ The private information exchange is assumed to take the form of coarsened communication. ${ }^{6}$

This paper should not be interpreted as contradicting the literature that stresses structural factors as root causes for a revolution (Skocpol 1979). Structural factors, such as the state of the economy and international pressure, make a society "ripe"

\footnotetext{
${ }^{3}$ Gentzkow and Shapiro (2006) show that individuals tend to believe that a news source is of high quality if it conforms to their prior expectations. Also see Suen (2010) for a model with similar features.

${ }^{4} \mathrm{~A}$ common implicit assumption in the literature is that citizens believe the public signal is informative. They assign a constant weight on the public signal based on its relative precision to private signals, and would not adjust the weight even though the public signal is remarkably different from what their own private information suggests.

${ }^{5}$ Angeletos and Werning (2006) explicitly acknowledge the importance of direct interaction between agents in coordination models. They allow agents to observe a public signal about the aggregate attack, which conveniently approximates the situation where agents could learn about the actions of others.

${ }^{6}$ Information coarsening is a cost-effective way of exchanging casual information, and is commonly used in real life.
} 
for revolution, but are not sufficient for the outbreak of a successful revolt. In line with Bueno de Mesquita (2010), we argue that some random factors also play a role in determining the fate of a revolution. In our model, the realization of rumors serves as a source of randomness.

Our work is related to a small economics literature on rumors, e.g., Banerjee (1993) and Bommel (2003). Unlike their models, in which a rumor is passed on to others sequentially, we provide a model in a static setting, in which a rumor is heard by citizens simultaneously. We focus on the effect of communication among citizens about the rumor rather than the transmission of the rumor itself.

This paper also contributes to a growing literature on revolutions in economics. Edmond (2011) considers a coordination game where citizens' private information about the regime's strength is contaminated by the regime's propaganda. Our model differs in that private information is uncontaminated, but the public signal may be false and unrelated to fundamentals. Both Bueno de Mesquita (2010) and Angeletos and Werning (2006) study coordination games with two stages, where public signals arise endogenously in the first stage. In our model, the "attack stage" is preceded by a "communication stage," where a private message endogenously arises and enlarges citizens' information set.

In other fields of social sciences, there is no lack of discussions on rumors (e.g., Allport and Postman 1947) and revolutions (e.g., Goldstone 1994). However there are few studies on the relationship between these two. The idea seems to have been "up in the air" that rumors motivate citizens to participate in social movements, but the precise mechanisms remain unspecified. Our model is a step toward formalizing one such mechanism to explain explicitly how rumors affect citizens' beliefs, actions, and therefore equilibrium outcomes in revolutions.

\section{A Model of Rumors and Talk about Rumors}

\subsection{Players and payoffs}

Consider a society populated by a unit mass of citizens, indexed by $i \in[0,1]$. Citizen $i$ chooses one of two actions: revolt $\left(a_{i}=1\right)$ or not revolt $\left(a_{i}=0\right)$. The aggregate mass of population that revolt is denoted $A$. Nature selects the strength of the regime, $\theta$, which is sometimes also referred to as the state. The regime survives if and only if $\theta>A$; otherwise it is overthrown. A citizen's payoff depends both on whether the regime is overthrown and on whether she chooses to revolt. A cost $c \in(0,1)$, has to be paid if she revolts. If the regime is overthrown, citizens who revolt receive a benefit 
$b=1$, and those who do not participate receive no benefit. ${ }^{7}$ A citizen's net utility is therefore:

$$
u\left(a_{i}, A, \theta\right)= \begin{cases}1-c, & \text { if } a_{i}=1 \text { and } A \geq \theta ; \\ -c, & \text { if } a_{i}=1 \text { and } A<\theta \\ 0, & \text { if } a_{i}=0 .\end{cases}
$$

\subsection{Information structure}

Citizens are ex ante identical and have improper prior on $\theta$. They become ex post heterogeneous after each of them observes a noisy private signal,

$$
x_{i}=\theta+\varepsilon_{i}
$$

where $\varepsilon_{i} \sim \mathcal{N}\left(0, \sigma_{x}^{2}\right)$ is independent of $\theta$ and is independently distributed across $i$. This assumption captures the situation that citizens have diverse assessments of the regime's strength before they hear any rumor and communicate. This seemingly standard assumption in the global games literature turns out to be crucial for our model, because citizens will not exchange information if they share the same belief.

All citizens hear a rumor, $z$, concerning the strength of the regime. The key issue that we focus on is how citizens evaluate and react to the rumor, when the rumor could be totally uninformative. Toward this end, the rumor is modeled as a public signal which may come from two alternative sources: either a source which offers an informative signal on the strength of the regime, or a source which only produces uninformative noise. Formally we model the random variable $z$ as coming from a mixture distribution:

$$
z \sim \begin{cases}I=\mathcal{N}\left(\theta, \sigma_{z}^{2}\right) & \text { with probability } \alpha \\ U=\mathcal{N}\left(s, \sigma_{U}^{2}\right) & \text { with probability } 1-\alpha\end{cases}
$$

where $I$ indicates the informative source and $U$ indicates the uninformative source. We assume that $\alpha, s, \sigma_{z}$, and $\sigma_{U}$ are commonly known to all citizens.

The parameter $s$ can be interpreted as the "sentiment" of the public, which captures their perception of what uninformative messages would sound like. ${ }^{8}$ For example, if

\footnotetext{
${ }^{7}$ We abstract from free-riding issues, which have been carefully addressed by Edmond (2011) and Bernhardt and Shadmehr (2011). The benefits from regime change can be modeled as a public good that all citizens would enjoy. Edmond (2011) offers a general payoff structure to accommodate this concern. He shows that a condition can be derived such that citizens still have incentives to act against the regime, despite the free-riding problem. To avoid being repetitive, we adopt a simpler payoff structure in this paper.

${ }^{8}$ The assumption that $s$ is common to all the citizens is made to simplify the exposition. Allowing them to possess diverse sentiments will not change our results.
} 
the public is used to receiving propaganda materials telling that the regime is strong, then they may expect a high value of $s$.

We stress that our specification of rumor as a mixture distribution is different from an informative public signal with low precision. According to the linear updating formula, all citizens would react to an informative public signal in the same way regardless of their private information. In our specification, however, citizens make an inference on the informativeness of the rumor based on their own private information and therefore have diverse opinions on the same rumor and react to it differently.

While not dismissing the relevance of rumormongers, we choose to model rumors as exogenous public signals in order to focus on their role in coordinating collective action. The origin and the content of rumors are assumed to be exogenous because of their sheer diversity and unpredictability. The possibility that rumors are cooked up strategically to influence individuals' beliefs is an important reason that people tend to be skeptical. But even in this case, the true sources of rumors are often shrouded in obscurity, making it difficult to infer whether they are manufactured to defend the regime or to destabilize it. ${ }^{9}$ Moreover, studies also show that rumors could be created unintentionally. For example, misunderstanding between individuals is a usual source of rumors (Allport and Postman 1947; Peterson and Gist 1951; Buchner 1965).

By modeling rumors as a public signal, we also abstract from the process of how rumors travel from one to another. ${ }^{10}$ It is implicitly assumed that rumors can reach every citizen in the game. ${ }^{11}$ This assumption seems to be realistic for many revolutions in history: rumors against authorities did gain a substantial, even huge, amount of publicity under very repressive regimes. ${ }^{12}$

We maintain the following parameter restrictions throughout this paper:

$$
\begin{aligned}
& \sigma_{x}<\sigma_{z}^{2} \sqrt{2 \pi} \\
& \sigma_{U}^{2}>\sigma_{x}^{2}+\sigma_{z}^{2} \equiv \sigma_{I}^{2} .
\end{aligned}
$$

The first restriction is standard. When $\alpha=1$, the model reduces to the standard global game model with public signal. Condition (1) is sufficient and necessary for

\footnotetext{
${ }^{9}$ See Knapp (1944), Nkpa (1977), Elias and Scotson (1994), and Gambetta (1994) for related analysis. The incentives of rumormongers could be unpredictable in the sense that they might be motivated by many different reasons (Zhang 2009; and Turner, et al. 1992).

${ }^{10}$ Acemoglu, Ozdaglar, and ParandehGheibi (2010) model how rumors spread in a network.

${ }^{11}$ We can also assume that a certain fraction of citizens do not hear any rumor, without affecting the main results in our model.

${ }^{12}$ The rumor that a student was killed by the police, which ignited the Velvet Revolution in Czechoslovakia, was broadcasted by Radio Free Europe. In 2009, the Iranian post-election protest intensified after a rumor surfaced in the internet that police helicopters poured acid and boiling water on protesters (Esfandiari 2010).
} 
the uniqueness of equilibrium in that model; see Angeletos and Werning (2006).

The second restriction captures the idea that uninformative noise exhibits greater variability than an informative signal. This assumption is motivated by the fact that an informative signal is generated based on the true strength of the regime and its realization is anchored by the truth, while there are multiple possibilities that can generate an uninformative signal. Rumors may be made up by friends or enemies of the regime, or by people with unknown motives that are unrelated to regime survival. The possibility that rumors are often the result of mistakes also adds to this uncertainty. In other words, since uninformative noise is drawn from a distribution, which is not anchored by facts or fundamentals, it tends to be more unpredictable. In Section 3, we show that this assumption is responsible for the result that citizens tend to discount wild rumors.

\subsection{Communication}

After citizens observe their private signals and the rumor, they are randomly paired up to communicate with each other about the informativeness of the rumor. Specifically each citizen in a pair expresses her view on the likelihood that the rumor is drawn from an informative source, and hears her peer's view on the same matter. We assume that citizens can only convey their views in a binary fashion. Let $y_{i}$ represent the message sent to citizen $i$ by her peer $k$. The communication technology is characterized as follows:

$$
y_{i}= \begin{cases}1, & \text { if } \operatorname{Pr}\left[z \sim I \mid z, x_{k}\right] \geq \delta \\ 0, & \text { if } \operatorname{Pr}\left[z \sim I \mid z, x_{k}\right]<\delta .\end{cases}
$$

The parameter $\delta$ is common to all citizens and can be interpreted as their threshold for plausibility. Citizen $k$ who sends the message $y_{i}=1$ to citizen $i$ can be interpreted as saying, "I believe the rumor is informative;" while the message $y_{i}=0$ can be interpreted as "I don't believe it." A high value of $\delta$ means that citizens are unlikely to say they believe in the rumor unless they are sufficiently confident of their assessments. To rule out the possibility that citizens will never say they believe in the rumor, we impose an upper bound for the value of $\delta$. Specifically, we assume:

$$
\delta<\frac{\alpha \sigma_{I}^{-1}}{\alpha \sigma_{I}^{-1}+(1-\alpha) \sigma_{U}^{-1}} \equiv \bar{\delta} .
$$

The communication rule (3) is non-strategic. Given that it is a game with a continuum of citizens, and that each citizen only talks to one other citizen, there is no incentive for citizens to strategically manipulate their peers' beliefs by lying. In a sense, truthful revelation is probably a good description of the casual communication between acquaintances. However, we also acknowledge that people may have other 
concerns when they communicate, and as a result they may behave strategically. In Section 5.2, we study a model of strategic communication and show that the key features of the communication rule in the non-strategic setting still hold.

To simplify our analysis, we assume that conversations between citizens are conducted in a binary fashion. However, what drives our results is not information coarsening, but the assumption that citizens talk about the informativeness of the rumor. The main insights of our model continue to hold if we allow people to exchange the exact values of their probability assessment. Rumors usually anchor the topic of conversations, especially when people take collective action. It is reasonable to assume that people are interested in their veracity and are inclined to discuss them. A model which allows for exchanging private signals only, without mentioning the rumor, will deliver very different results. Section 5.1 elaborates on the role of alternative communication protocols in our model.

\subsection{Posterior beliefs, decision rules and equilibrium}

This model can be analyzed in two stages. In the communication stage, citizen $i$ sends a message, $y_{k} \in\{0,1\}$, to her peer $k$ based on the information set $\left\{z, x_{i}\right\}$, and receives a private message $y_{i} \in\{0,1\}$ from her peer $k$. In the attack stage, citizen $i$ chooses to revolt or not, given the post-communication information set $\left\{z, x_{i}, y_{i}\right\}$, to maximize her expected utility.

Before communication, citizens update their beliefs on the mixture weight:

$$
\operatorname{Pr}\left[z \sim I \mid z, x_{i}\right]=\frac{\alpha \sigma_{I}^{-1} \phi\left(\sigma_{I}^{-1}\left(z-x_{i}\right)\right)}{\alpha \sigma_{I}^{-1} \phi\left(\sigma_{I}^{-1}\left(z-x_{i}\right)\right)+(1-\alpha) \sigma_{U}^{-1} \phi\left(\sigma_{U}^{-1}(z-s)\right)} \equiv w\left(z, x_{i}\right),
$$

where $\phi$ is the standard normal density function. The function $w\left(z, x_{i}\right)$ is singlepeaked in $x_{i}$, reaching the maximum at $x_{i}=z$. This means that a citizen is more likely to believe the rumor is informative if her private information is closer to the rumor. There exists $\underline{x}(z)$ and $\bar{x}(z)$ such that $w(z, \underline{x}(z))=w(z, \bar{x}(z))=\delta$. Therefore, the communication decision rule can be written as:

$$
y\left(z, x_{i}\right)= \begin{cases}1, & \text { if } x_{i} \in[\underline{x}(z), \bar{x}(z)] \\ 0, & \text { otherwise }\end{cases}
$$

After communication, citizens update their beliefs with the message $y_{i}$ received from their peers. Their beliefs will be re-weighted by the likelihood of receiving $y_{i}$ for each state. Let $P\left(\cdot \mid z, x_{i}, y_{i}\right)$ be the cumulative distribution of the state, with the corresponding posterior density $p\left(\cdot \mid z, x_{i}, y_{i}\right)$. By Bayes' rule, citizen $i$ who receives the 


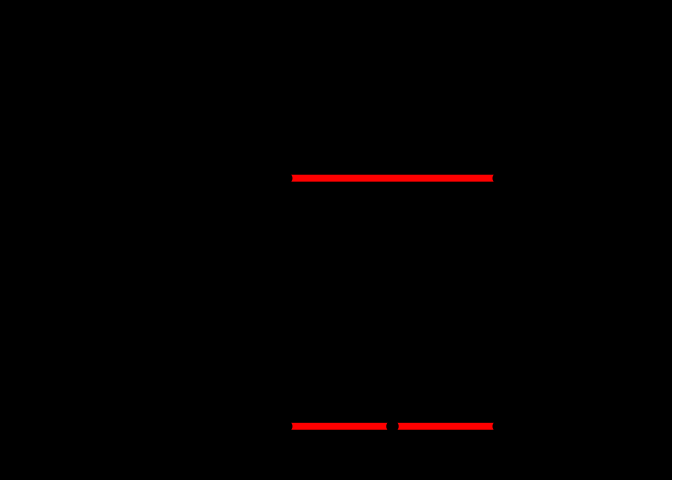

Figure 1. Citizens with $x_{i} \in[\underline{x}(z), \bar{x}(z)]$ say they believe the rumor. In the aggregate, the mass of such citizens is $J(\theta, z)$.

message $y_{i}=1$ from her peer revises her belief about the state to:

$$
P\left(\theta \mid z, x_{i}, 1\right)=\frac{\int_{-\infty}^{\theta} J(t, z) p\left(t \mid z, x_{i}\right) \mathrm{d} t}{\int_{-\infty}^{\infty} J(t, z) p\left(t \mid z, x_{i}\right) \mathrm{d} t} .
$$

In this formula, $p\left(\cdot \mid z, x_{i}\right)$ is the density associated with the belief $P\left(\cdot \mid z, x_{i}\right)$ before communication, and $J(t, z)$ is the probability of receiving message $y_{i}=1$ in state $t$ :

$$
J(t, z)=\Phi\left(\frac{\bar{x}(z)-t}{\sigma_{x}}\right)-\Phi\left(\frac{\underline{x}(z)-t}{\sigma_{x}}\right)
$$

where $\Phi$ is the standard normal distribution function. See Figure 1 for illustration. Similarly, a citizen $i$ who receives the message $y_{i}=0$ revises her belief to:

$$
P\left(\theta \mid z, x_{i}, 0\right)=\frac{\int_{-\infty}^{\theta}(1-J(t, z)) p\left(t \mid z, x_{i}\right) \mathrm{d} t}{\int_{-\infty}^{\infty}(1-J(t, z)) p\left(t \mid z, x_{i}\right) \mathrm{d} t} .
$$

In this paper, we focus on monotone equilibrium in which, for any $z$, there is a survival threshold $\theta^{*}(z)$ such that the regime collapses if and only if $\theta \leq \theta^{*}(z)$. For any fixed regime survival threshold $\hat{\theta}$, the expected payoff from attacking the regime given the information set $\left\{z, x_{i}, y_{i}\right\}$ decreases in $x_{i}$, because the posterior belief about $\theta$ is stochastically increasing in $x_{i}$ (Milgrom 1981). Thus, there exist unique cutoff types $\hat{x}_{I}$ and $\hat{x}_{U}$ such that the indifference conditions hold:

$$
\begin{aligned}
P\left(\hat{\theta} \mid z, \hat{x}_{I}, 1\right) & =c \\
P\left(\hat{\theta} \mid z, \hat{x}_{U}, 0\right) & =c .
\end{aligned}
$$

Citizen $i$ chooses to attack the regime when $x_{i} \leq \hat{x}_{I}$ and $y_{i}=1$, or when $x_{i} \leq \hat{x}_{U}$ and $y_{i}=0$. 
Let $A\left(\theta ; \hat{x}_{I}, \hat{x}_{U}, z\right)$ be the mass of attackers when the state is $\theta$ and when citizens adopt the cutoff rules $\hat{x}_{I}$ and $\hat{x}_{U}$ :

$$
A\left(\theta ; \hat{x}_{I}, \hat{x}_{U}, z\right)=J(\theta, z) \Phi\left(\frac{\hat{x}_{I}-\theta}{\sigma_{x}}\right)+(1-J(\theta, z)) \Phi\left(\frac{\hat{x}_{U}-\theta}{\sigma_{x}}\right)
$$

The regime survival threshold must satisfy the critical mass condition,

$$
A\left(\hat{\theta} ; \hat{x}_{I}, \hat{x}_{U}, z\right)=\hat{\theta}
$$

A monotone equilibrium is characterized by a triple $\left(\theta^{*}(z), x_{I}^{*}(z), x_{U}^{*}(z)\right)$ that solves equations (8), (9) and (10).

\section{Rumors without Communication}

Our model departs from the standard global game model in two respects: (1) the public signal may be uninformative; and (2) citizens can exchange messages concerning the informativeness of the public signal. To highlight the effects of these two features separately, we discuss in this section a model with feature (1) only by setting $\delta=0$, so that everyone always sends the same message, and communication becomes irrelevant. We refer to this special case as the "mute model," and let $\left(\theta_{m}^{*}, x_{m}^{*}\right)$ represent the equilibrium regime survival threshold and cutoff type.

The "mute model" nests two important benchmarks. When $\alpha=0$, citizens believe that the rumor is completely uninformative. We label it the "pure noise model" and use $\left(\theta_{m s}^{*}, x_{m s}^{*}\right)$ to denote the equilibrium pair. When $\alpha=1$, the rumor is known to be trustworthy. We refer to this case as the "public signal model" and use $\left(\theta_{p s}^{*}, x_{p s}^{*}\right)$ to denote the equilibrium pair. In this case, $\theta_{p s}^{*}(z)$ monotonically decreases in $z .{ }^{13}$

In the "mute model," the posterior belief about $\theta$ upon hearing a rumor $z$ is a mixture of the posterior distribution in the "public signal model" and that in the "pure noise model," with weights given by the posterior belief that the rumor is informative or not. In other words,

$$
P\left(\theta \mid z, x_{i}\right)=w\left(z, x_{i}\right) \Phi\left(\frac{\theta-X_{i}}{\sqrt{\beta} \sigma_{x}}\right)+\left(1-w\left(z, x_{i}\right)\right) \Phi\left(\frac{\theta-x_{i}}{\sigma_{x}}\right)
$$

where citizen $i$ 's posterior mean upon observing an informative public signal is $X_{i}=$ $\beta x_{i}+(1-\beta) z$ with $\beta=\sigma_{z}^{2} /\left(\sigma_{z}^{2}+\sigma_{x}^{2}\right)$, and the posterior variance is $\beta \sigma_{x}^{2}$. Since communication is ineffective, the critical mass condition reduces to the standard one.

Lemma 1. There exists a unique neutral rumor $z^{\prime}$ such that $\theta_{m}^{*}\left(z^{\prime}\right)=\theta_{p s}^{*}\left(z^{\prime}\right)=\theta_{m s}^{*}$

\footnotetext{
${ }^{13}$ The subscript $m s$ stands for "Morris-Shin," $p$ s for "public signal," and $m$ for "mute."
} 
and $\theta_{p s}^{*}(z)>\theta_{m s}^{*}$ for all $z<z^{\prime}$ and $\theta_{p s}^{*}(z)<\theta_{m s}^{*}$ for all $z>z^{\prime}$.

The proof of this and other technical lemmas is in the Technical Appendix. In what follows, we say that a rumor is against the regime or negative if $z<z^{\prime}$, and that the rumor is for the regime or positive if $z>z^{\prime}$. Further, we say that a negative rumor is moderate if $z$ is slightly below $z^{\prime}$, and is extreme if $z$ approaches negative infinity. In the "mute model," upon hearing the neutral rumor $z^{\prime}$, the cutoff citizen is indifferent as to whether the rumor is informative or not, because the likelihood of success is the same in both cases. This is why the equilibrium pair $\left(\theta_{m s}^{*}, x_{m s}^{*}\right)$ of the "pure noise model" also solves the "mute model" when $z=z^{\prime}$.

Skepticism is the key feature of the "mute model": citizens take into account the possibility that the rumor could just be uninformative noise. Skepticism towards rumors is manifest in three aspects.

Proposition 1. (a) Extreme rumors have no impact, i.e., $\lim _{z \rightarrow \pm \infty} \theta_{m}^{*}(z)=\theta_{m s}^{*}$. (b) For any $z, \theta_{m}^{*}(z)$ is between $\theta_{m s}^{*}$ and $\theta_{p s}^{*}(z)$. (c) $\theta_{m}^{*}(z)$ is non-monotone in $z$.

If a rumor in circulation is extreme, almost all citizens consider it to be uninformative noise because it is so far from their own private information. Therefore, the equilibrium is identical to that in the "pure noise model."14

The effect of skepticism also manifests itself in the fact that $\theta_{m}^{*}(z)$ is between $\theta_{m s}^{*}$ and $\theta_{p s}^{*}(z)$ for any $z$. In the "mute model," citizens are less responsive to the rumor than when they are sure that it is informative, but are more responsive to it than when they are sure that it is not. This, however, does not mean that the "mute model" is simply a "public signal model" with a less precise public signal.

In fact, an interesting aspect of skepticism is that $\theta_{m}^{*}(z)$ is not monotonic, in contrast to the monotonicity of $\theta_{p s}^{*}(z)$. To see the intuition, observe that from equation (11),

$$
\frac{\partial P\left(\theta_{m}^{*} \mid z, x_{i}\right)}{\partial z}=-w \frac{\beta}{\sqrt{\beta} \sigma_{x}} \phi_{I}+\left(\Phi_{I}-\Phi_{U}\right) \frac{\partial w}{\partial z},
$$

where the subscripts $I$ and $U$ mean that the functions are evaluated at the points $\left(\theta_{m}^{*}-\right.$ $\left.X_{i}\right) /\left(\sqrt{\beta} \sigma_{x}\right)$ and $\left(\theta_{m}^{*}-x_{i}\right) / \sigma_{x}$, respectively. If the rumor is informative, an increase in $z$ is an indication that the regime is strong, which lowers the probability that the regime will collapse. Hence, the first term is negative. This is the standard "public signal effect." The second term captures the "skepticism effect," which is positive for

\footnotetext{
${ }^{14}$ This property follows from our the parameter restriction (2), under which, for any finite $x_{i}, w\left(z, x_{i}\right)$ approaches 0 when $z$ is extreme. When $\sigma_{I}<\sigma_{U}$, an extreme $z$ is infinitely more likely to be generated from an uninformative source than an informative one. But if we assume the opposite, $w\left(z, x_{i}\right)$ has a single trough, and approaches 1 when $z$ is extreme. In that case, citizens would regard rumors which are further away from their private information to be more plausible.
} 
extreme values of $z$. For instance, when $z$ is sufficiently large, $\partial w / \partial z<0$, which reflects greater skepticism toward the rumor when it moves further away from the citizen's private signal. Moreover, $\Phi_{I}-\Phi_{U}<0$, because the probability that the regime will collapse is lower if a rumor for the regime is informative than if it is not.

For $z$ sufficiently close to $z^{\prime}$, the magnitude of $\Phi_{I}-\Phi_{U}$ is small, because it does not matter to the cut-off type whether the neutral rumor is informative or not. Therefore, the "public signal effect" dominates and the survival threshold decreases in $z$ : a rumor suggesting a moderately stronger regime discourages mobilization. For $z$ sufficiently extreme, the magnitude of the "public signal effect" is small but that of the "skepticism effect" is large. When the second term dominates the first term, a wild rumor suggesting a very strong regime raises doubt about its veracity and may actually encourage mobilization. ${ }^{15}$

\section{Rumors with Communication}

In this section, we let $\delta \in(0, \bar{\delta})$ so that there is meaningful communication among the citizens. We focus on three key results in this "communication model." First, communication makes citizens even more skeptical of extreme rumors. When an extreme rumor against the regime circulates, communication discourages attackers. Second, communication makes citizens more responsive to rumors that are close to neutral. When a rumor moderately against the regime circulates, communication encourages attackers and can cause the regime to collapse, even though it could have survived had citizens remained silent. Third, the effect of communication can be so potent that a rumor can mobilize even more attackers than when the same story is regarded by everybody as fully trustworthy.

In the analysis of rumors that are close to neutral, we rely on an interesting special case where $c=0.5$. In this case, the cutoff type under all the four types of information structure are the same when the neutral rumor is in circulation. In other words, neutral rumors are not only those that cutoff type citizens do not care about from which source they are drawn $(z \sim I$ or $z \sim U)$, but also those that they do not care about what their peers say $(y=1$ or $y=0)$. As a result, equilibrium thresholds are also the same across all the four models when $z=z^{\prime}$. We study the role of communication by letting $z$ deviate from $z^{\prime}$ and comparing the equilibrium results with the other three benchmarks. This special case offers tractability for our analysis and facilitates the exposition of how communication matters for the equilibrium outcomes. We discuss the case where $c \neq 0.5$ in Section B of the Technical Appendix and show that the

\footnotetext{
${ }^{15}$ The properties of $x_{m}^{*}(z)$ are similar to those of $\theta_{m}^{*}(z)$ : it increases then decreases and then increases in $z$. This follows from the critical mass condition, which can be written as $x_{m}^{*}(z)=\theta_{m}^{*}(z)+$ $\sigma_{x} \Phi^{-1}\left(\theta_{m}^{*}(z)\right)$. Under parameter restriction $(1), x_{m}^{*}(z)$ is increasing in $\theta_{m}^{*}(z)$.
} 
qualitative properties are the same.

\subsection{Communication and Aggressiveness}

Proposition 2. Suppose $c=0.5$. (a) If the rumor is neutral, communication has no effect on the decision rule of the citizens, i.e., $x_{I}^{*}\left(z^{\prime}\right)=x_{U}^{*}\left(z^{\prime}\right)=x_{m}^{*}\left(z^{\prime}\right)$. (b) If the rumor is against the regime, citizens are more aggressive in attacking when receiving a confirmatory message than when receiving a contradictory message; i.e., $x_{I}^{*}(z)>x_{U}^{*}(z)$ for all $z<z^{\prime}$. Similarly, $x_{I}^{*}(z)<x_{U}^{*}(z)$ for all $z>z^{\prime}$.

When a negative rumor is in circulation, citizens with private information $x_{i}<x_{U}^{*}$ attack the regime regardless of the message they receive. We label this group of citizens revolutionaries. Citizens with private information $x_{i}>x_{I}^{*}$ do not revolt regardless. This group is labeled bystanders. The group of citizens with $x_{i} \in\left[x_{U}^{*}, x_{I}^{*}\right]$ is called the swing population. Their revolt decisions are influenced by their peers' assessments of the rumor: they choose to revolt if they receive $y_{i}=1$, and not to revolt otherwise. To see why citizens from the swing population become more aggressive when receiving a confirmatory message of a negative rumor, we need the following lemma.

Lemma 2. At $z=z^{\prime}$ and $\hat{\theta}=\theta^{*}\left(z^{\prime}\right)$, the cutoff types who are indifferent between attacking and not attacking satisfy:

$$
\begin{gathered}
\frac{\partial \hat{x}_{I}}{\partial z}<\frac{\partial \hat{x}_{m}}{\partial z}<\frac{\partial \hat{x}_{U}}{\partial z}<0, \\
\frac{\partial \hat{x}_{I}}{\partial \hat{\theta}}>\frac{\partial \hat{x}_{m}}{\partial \hat{\theta}}>\frac{\partial \hat{x}_{U}}{\partial \hat{\theta}}>0 .
\end{gathered}
$$

When the rumor is neutral, the cutoff citizen is indifferent between receiving $y=1$ or $y=0$ from her peer, i.e., $x_{I}^{*}=x_{U}^{*}=x_{m}^{*}$. The ordering of $\partial \hat{x} / \partial z$ (direct effect) implies that a citizen who receives $y=1$ reacts more to the rumor than she does without such communication. ${ }^{16}$ The reason is that a confirmatory message $(y=1)$ causes a citizen to believe that states close to the rumor are more likely to be the true strength of the regime. In other words, the message leads to a more concentrated posterior density for states around $\theta=z^{\prime}$. This effect can be seen from equation (6), which implies that

$$
\frac{p\left(\theta \mid z^{\prime}, x, 1\right)}{p\left(\theta \mid z^{\prime}, x\right)}=\frac{J\left(\theta, z^{\prime}\right)}{\int_{-\infty}^{\infty} J\left(t, z^{\prime}\right) p\left(t \mid z^{\prime}, x\right) \mathrm{d} t} .
$$

The likelihood $J\left(\theta, z^{\prime}\right)$ in the numerator is increasing then decreasing in $\theta$, with a peak

\footnotetext{
${ }^{16}$ The direct effect comes from the response of individuals, without taking into account the resulting change in equilibrium survival threshold, and its magnitude depends on $\partial \hat{x} / \partial z$.
} 
at $\theta=z^{\prime}$. The denominator is just the expected value of $J\left(\theta, z^{\prime}\right)$. Therefore, the ratio in (15) is greater than 1 when $\theta$ is close to $z^{\prime}$ and smaller than 1 when $\theta$ is far from it. Moreover, the same increase in the value of $z$ shifts the density function $p\left(\cdot \mid z, x_{i}, 1\right)$ to the right by a greater amount than it does to the density function $p\left(\cdot \mid z, x_{i}\right)$. This explains why, in response to the same amount of change in $z, \hat{x}_{I}$ has to decrease by a larger amount than $\hat{x}_{m}$ in order to restore the indifference condition.

Consider next the ordering of $\partial \hat{x} / \partial \hat{\theta}$ (multiplier effect). ${ }^{17}$ When $c=0.5, J\left(\theta, z^{\prime}\right)$ reaches a peak at $\theta=\theta^{*}\left(z^{\prime}\right)$. Thus, equation (15) implies that $p\left(\theta^{*}\left(z^{\prime}\right) \mid z^{\prime}, x, 1\right)>$ $p\left(\theta^{*}\left(z^{\prime}\right) \mid z^{\prime}, x\right)$. The term $p\left(\theta^{*}\left(z^{\prime}\right) \mid z^{\prime}, x\right)$ reflects the increase in payoff from attacking in the "mute model" when the regime survival threshold is marginally raised above $\theta^{*}\left(z^{\prime}\right)$. Since raising the survival threshold increases the incentive to attack by a greater amount for citizens who receive a confirmatory message, $\hat{x}_{I}$ also has to increase by a greater amount than $\hat{x}_{m}$ does to keep the cutoff types indifferent.

\subsection{Does Communication Help or Hurt?}

The goal of our analysis is to shed light on why some rumors can be so effective while others are not. We show that communication among citizens is the key with the following two results: communication encourages mobilization when the rumor is moderately negative, but it discourages mobilization when the rumor is extremely negative. ${ }^{18}$ See Figure 2 for a comparison of the regime survival thresholds under the "communication model" and the "mute model."19 Interestingly, these two contrasting results are driven by the same mechanism. To explain, we start with how communication affects the total mass of attackers and then elaborate on how it translates into the ranking of equilibrium thresholds.

Communication among citizens allows them to be better informed regarding where $\theta$ lies. They know better about whether $\theta$ is close to or far away from $z$ than their counterparts in the "mute model." The key is that the fraction of citizens who send confirmatory messages, $J(\theta, z)$, is larger if the regime strength is closer to what the

\footnotetext{
${ }^{17}$ The multiplier effect arises because of the complementarity in action among citizens, which amplifies the direct effect: when citizens become more aggressive, the regime survival threshold has to increase, which in turn raises the payoff from revolting and hence further increases the cutoff type. The magnitude of the multiplier effect depends on $\partial \hat{x} / \partial \hat{\theta}$.

${ }^{18}$ An alternative question is which information structure-with or without communication-will be preferred by the regime ex ante, i.e., before the rumor is heard. In order to answer this question, we can compute the ex ante survival probability for each $\theta$, by integrating over $z$ using the mixture distribution. However, once we pool together all these effective and ineffective rumors as well as positive and negative rumors, the answer to this question depends on the parameters of the model and no general conclusion can be made.

${ }^{19}$ To compute the numerical examples in the figures, unless otherwise specified, we use the following set of parameters: $c=0.5, s=0.5, \alpha=0.5, \delta=0, \sigma_{U}^{2}=1, \sigma_{z}^{2}=0.5$, and $\sigma_{x}^{2}=0.4$ for the "pure noise model," "public signal model" and "mute model." For the "communication model," we set $\delta=0.5$.
} 


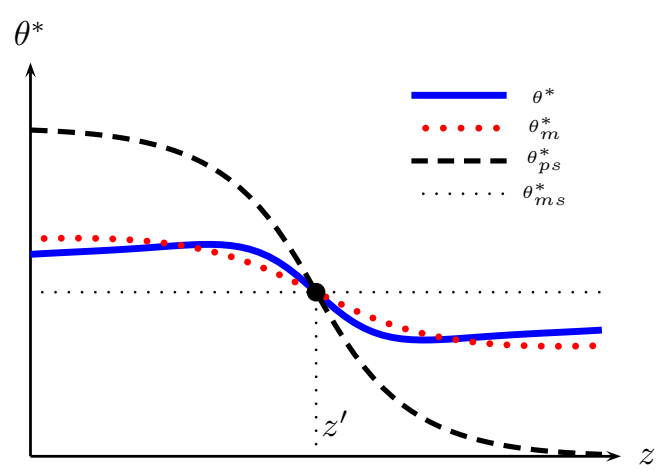

(a) regime survival thresholds

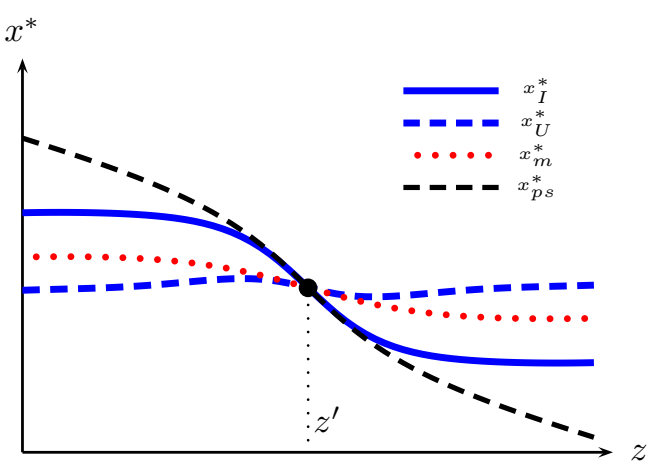

(b) cutoff agent types

Figure 2. Equilibrium regime survival thresholds and equilibrium cutoff citizen types in the "communication model" in comparison to other models.

rumor suggests, and achieves a maximum when $\theta=z$. Therefore, more citizens will hear confirmatory messages when $\theta$ is near $z$ and fewer when $\theta$ is far away from $z$.

Recall that citizens from the swing population revolt when receiving a confirmatory message of a negative rumor. If the rumor says that the regime is fragile and the regime is indeed weak, a larger fraction of the swing population will receive the message $y=1$ from their peers and decide to join the revolutionaries and attack the regime, which results in a larger total mass of attackers $A(\theta)$ in the "communication model." On the other hand, the total mass of attackers will be smaller if the true regime strength is very different from what the rumor says, because only a very small fraction of the swing population will attack the regime.

Figure 3 plots the mass of attackers $A(\theta)$ against the regime strength $\theta$, holding the cutoff rules constant. Figure 3(a) shows the situation for $z$ being slightly below $z^{\prime}$ : a larger mass of attackers are mobilized than that in the "mute model" when the regime strength $\theta$ is near $z$, and the mass is smaller when the true $\theta$ is far away from $z$. For the same reason, if the rumor indicates the regime is extremely fragile, it mobilizes more attackers when the regime is indeed extremely weak. But the mass of attackers falls relative to that in the "mute model" when the regime is indeed strong and far away from what the rumor suggests. Figure 3(b) illustrates this situation.

In Figure 3, the mass of attackers $A(\theta)$ in the "communication model" is larger than its counterpart in the "mute model" for states close to $z$. Since the equilibrium regime survival threshold is given by the intersection of $A(\theta)$ and the 45-degree line, this figure shows that $\theta^{*}(z)>\theta_{m}^{*}(z)$ for moderately negative $z$, and $\theta^{*}(z)<\theta_{m}^{*}(z)$ for extremely negative $z$.

Proposition 3. When an extreme negative rumor is in circulation, communication among citizens makes the regime less vulnerable to attacks, i.e., $\theta^{*}(z)<\theta_{m}^{*}(z)$. Similarly, $\theta^{*}(z)>$ 


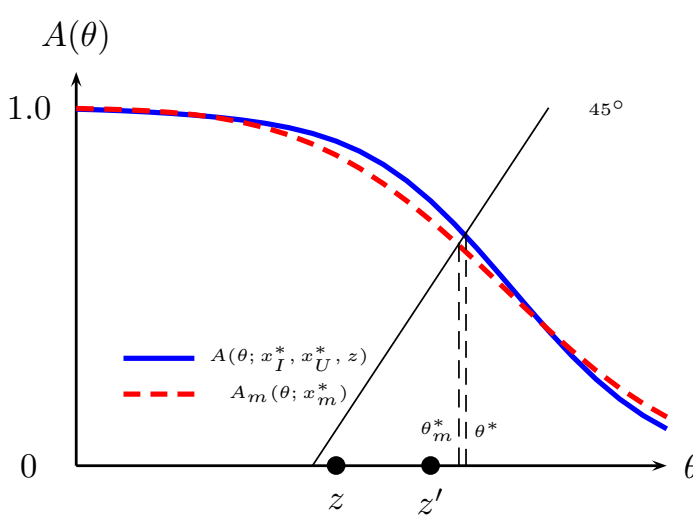

(a) A mild negative rumor, i.e., $z<z^{\prime}$

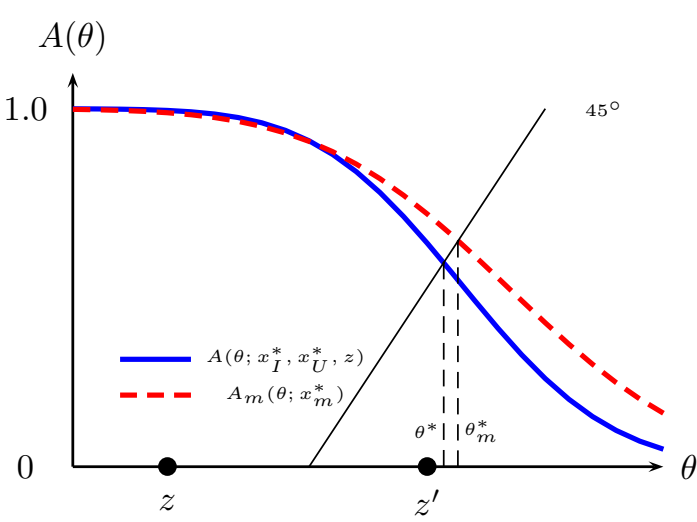

(b) An extreme negative rumor, i.e., $z<<z^{\prime}$

Figure 3. Communication increases the mass of attackers in states close to $z$ but lowers it in states far away from $z$.

$\theta_{m}^{*}(z)$ when $z$ is extremely positive.

Consider an extreme negative rumor. The cutoff citizens who receive $y=0$ will be less aggressive than the cutoff type in the "mute model," who is in turn less aggressive than the cutoff citizens receiving $y=1$. That is, $x_{U}^{*}<x_{m}^{*}<x_{I}^{*}{ }^{20}$ Because $z$ is extremely negative, unless the true regime strength is extremely weak, the fraction of citizens who receive confirmatory messages, $J(\theta, z)$, will be very small. Therefore, only a negligible fraction of the swing population will join the revolutionaries and attack the regime. As a result, the mass of attackers in the "communication model" is smaller than in the "mute model." That is,

$$
J(\theta, z)\left[\Phi\left(\frac{x_{I}^{*}-\theta}{\sigma}\right)-\Phi\left(\frac{x_{U}^{*}-\theta}{\sigma}\right)\right]+\Phi\left(\frac{x_{U}^{*}-\theta}{\sigma}\right)<\Phi\left(\frac{x_{m}^{*}-\theta}{\sigma}\right),
$$

or $A(\theta)<A_{m}(\theta) .{ }^{21}$ As a result, Proposition 3 is implied.

Proposition 4. When a moderately negative rumor is in circulation, communication among citizens makes the regime more vulnerable to attacks, i.e., $\theta^{*}(z)>\theta_{m}^{*}(z)$. Similarly, $\theta^{*}(z)<$ $\theta_{m}^{*}(z)$ when $z$ is moderately positive.

To understand this proposition, we study the change in total mass of attackers when $z$ deviates from $z^{\prime}$. In the "communication model," a slight decrease in $z$ from $z^{\prime}$ leads to an increase in the size of swing population by $\sigma_{x}^{-1} \phi(\cdot)\left(\mathrm{d} x_{I}^{*} / \mathrm{d} z-\mathrm{d} x_{U}^{*} / \mathrm{d} z\right)$. A fraction $J$ of the swing population would receive a confirmatory message from their peers and decide to join the revolutionaries and attack the regime. The change in the

\footnotetext{
${ }^{20}$ The first inequality is established in the proof of Lemma 8 , and the second follows from part 1 of Lemma 10.

${ }^{21}$ The proof of Proposition 3 shows that, as $z$ gets sufficiently negative, the first term of the lefthand-side varnishes much quicker than the difference between the second term and the term on the right-hand-side.
} 
mass of revolutionaries is $\sigma_{x}^{-1} \phi(\cdot) \mathrm{d} x_{U}^{*} / \mathrm{d} z$. In the "mute model," the increase in the mass of attackers is $\sigma_{x}^{-1} \phi(\cdot) \mathrm{d} x_{m}^{*} / \mathrm{d} z$. For communication to increase the size of attack given a moderate rumor against the regime, we must have

$$
\frac{1}{\sigma_{x}} \phi(\cdot)\left[J(\cdot)\left(\frac{\mathrm{d} x_{I}^{*}}{\mathrm{~d} z}-\frac{\mathrm{d} x_{U}^{*}}{\mathrm{~d} z}\right)+\frac{\mathrm{d} x_{U}^{*}}{\mathrm{~d} z}\right]<\frac{1}{\sigma_{x}} \phi(\cdot) \frac{\mathrm{d} x_{m}^{*}}{\mathrm{~d} z}<0 .
$$

To see why inequality (16) holds, it is sufficient to show that both direct and multiplier effects are larger in the "communication model" than those in the "mute model."22 That is, at the point $z=z^{\prime}$ and $\hat{\theta}=\theta^{*}\left(z^{\prime}\right)$,

$$
\begin{aligned}
& J \frac{\partial \hat{x}_{I}}{\partial z}+(1-J) \frac{\partial \hat{x}_{U}}{\partial z}<\frac{\partial \hat{x}_{m}}{\partial z}<0 \\
& J \frac{\partial \hat{x}_{I}}{\partial \hat{\theta}}+(1-J) \frac{\partial \hat{x}_{U}}{\partial \hat{\theta}}>\frac{\partial \hat{x}_{m}}{\partial \hat{\theta}}>1
\end{aligned}
$$

Inequalities (17) and (18) are stronger than Lemma 2. They hold because a large fraction of the population will receive confirmatory messages when the rumor is close to the true state. As a result, the population as a whole is better informed given the extra information from communication. Further, consistent with inequality (16), we establish in the proof that $\theta^{*}$ is more responsive to the change in $z$ around $z^{\prime}$ than is $\theta_{m}^{*}$, which therefore implies Proposition 4.

\subsection{The Power of Whispers: Rumors vs. Trustworthy News}

Strikingly, the effect of communication can be so large that, when a rumor against the regime circulates, the regime could survive when all citizens believe that it is trustworthy, but could collapse when citizens know that the rumor may be uninformative.

In this comparison, communication is also allowed in the "public signal model," but it is completely ineffective. Given everybody believes that the rumor is informative (i.e., $\alpha=1$ ), the posterior belief of the informativeness is always $w\left(z, x_{i}\right)=1$. Therefore, for any plausibility threshold $\delta$, everybody would send to and receive from her peers the message $y=1$. Paradoxically, communication takes no effect in the absence of skepticism.

Figure 4 shows that $\theta^{*}$ can be higher than $\theta_{p s}^{*}$ for $z$ slightly below $z^{\prime}{ }^{23}$ To see why this possibility could arise, we need to compare $A(\theta)$ with $A_{p s}(\theta)$ at $\theta=\theta^{*}\left(z^{\prime}\right)$ when

\footnotetext{
${ }^{22}$ We note that $\mathrm{d} x^{*} / \mathrm{d} z=\partial x^{*} / \partial z+\left(\partial x^{*} / \partial \hat{\theta}\right)\left(\mathrm{d} \theta^{*} / \mathrm{d} z\right)$. Therefore, the magnitude of $\mathrm{d} x^{*} / \mathrm{d} z$ depends on both direct and multiplier effects. At the aggregate level, the direct effect in the "communication model" is the weighted average of $\partial \hat{x}_{I} / \partial z$ and $\partial \hat{x}_{U} / \partial z$, with the weights being $J$ and $1-J$, respectively. The multiplier effect is the weighted average of $\partial \hat{x}_{I} / \partial \hat{\theta}$ and $\partial \hat{x}_{U} / \partial \hat{\theta}$.

${ }^{23}$ In plotting this figure, we set $\delta=0.5$ and use the value of $\sigma_{x}^{2}=0.2$, which is lower than that used in Figure 2(a).
} 


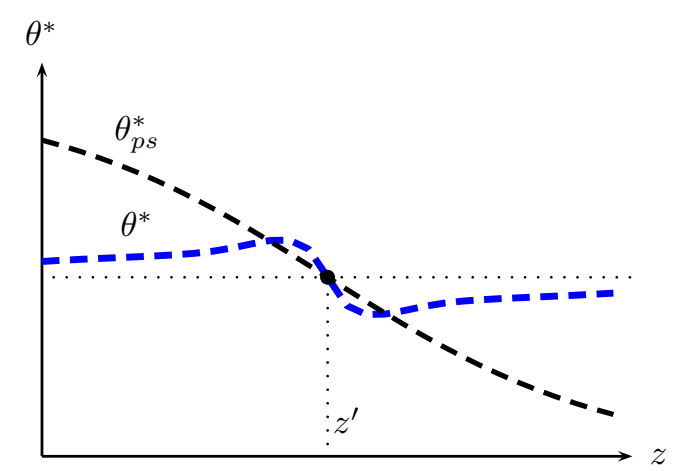

Figure 4. Comparing the "communication model" to the "public signal model" when private information has moderately high precision.

$z$ deviates from $z^{\prime}$ to a slightly more negative value. Similar to the previous analysis, the response of the mass of attackers to a change in $z$ is governed by the direct and multiplier effects. In the following analysis, we just focus on the multiplier effect, i.e., $\partial \hat{x} / \partial \hat{\theta}^{24}$

We have shown the following two mechanisms separately: due to the effect of skepticism, $\partial \hat{x}_{p s} / \partial \hat{\theta}>\partial \hat{x}_{m} / \partial \hat{\theta}$; and due to the effect of communication, $\partial \hat{x}_{I} / \partial \hat{\theta}>$ $\partial \hat{x}_{m} / \partial \hat{\theta}>\partial \hat{x}_{U} / \partial \hat{\theta}$. Interestingly, under some conditions, the fraction of population who receive confirmatory messages can be large, such that the average response of the population is larger than that in the "public signal model." That is, at the point $z=z^{\prime}$ and $\hat{\theta}=\theta^{*}\left(z^{\prime}\right)$,

$$
J \frac{\partial \hat{x}_{I}}{\partial \hat{\theta}}+(1-J) \frac{\partial \hat{x}_{U}}{\partial \hat{\theta}}>\frac{\partial \hat{x}_{p s}}{\partial \hat{\theta}} .
$$

This result is interesting in that skepticism provides the ground for communication, but its effect can also be undone by communication.

Proposition 5. Suppose the relative precision $\beta$ of private information is not too low, and the threshold $\delta$ for sending a confirmatory message is neither too high nor too low. A regime is more vulnerable to a moderately negative rumor $z$ when citizens believe that it could be false than when they consider it fully trustworthy, i.e., $\theta^{*}(z)>\theta_{p s}^{*}(z)$.

To understand Proposition 5, note that communication is not very informative when the value of $\delta$ is very large or very small, because most citizens would send the same message. Figure 5 shows that the multiplier effect in the "communication model" (the left-hand-side of inequality (19)) is the same as the multiplier effect in the "mute model" when $\delta$ is close to its bounds. Figure 5 also shows that the effect of communication is hump-shaped: for intermediate values of $\delta$, the multiplier effect in the "communication model" is larger than that in the "mute model," and can even exceed

\footnotetext{
${ }^{24}$ Lemma 6 of the Technical Appendix establishes that the direct and multiplier effects sum up to 1 . A stronger multiplier effect implies that the direct effect is also stronger.
} 


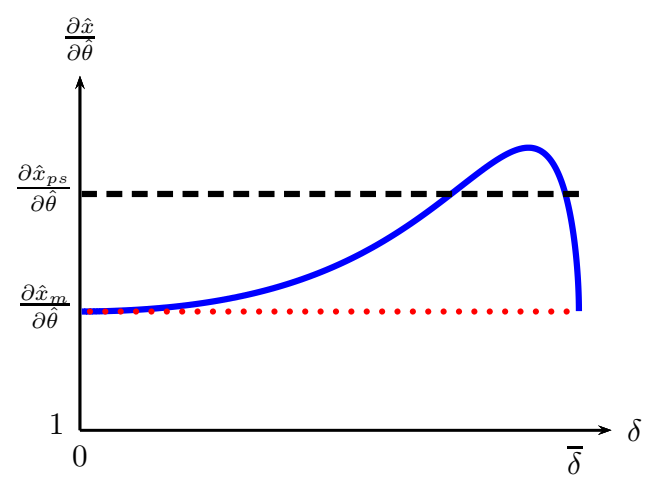

Figure 5. The hump-shaped communication effect. The solid curve stands for the multiplier effect in the "communication model;" the dashed and dotted lines represent the multiplier effect in the "public signal model" and "mute model," respectively.

that in the "public signal model."

Recall that $\delta$ determines the fraction of population $J$ who send confirmatory messages. When the plausibility threshold $\delta$ is low, $J$ is high, because citizens are less cautious in saying that they believe the rumor. Given that receiving a confirmatory message is so expected, recipients of such messages would not update their belief that much. Therefore, $\partial \hat{x}_{I} / \partial \hat{\theta}$ will be quite close to $\partial \hat{x}_{m} / \partial \hat{\theta}$.

When $\delta$ is larger, citizens become more careful about sending their peers confirmatory messages and such messages will be stronger evidence that $\theta$ is close to $z^{\prime}$. In this case, recipients of confirmatory messages can be even more responsive than those in the "public signal model," i.e., $\partial \hat{x}_{I} / \partial \hat{\theta}>\partial \hat{x}_{p s} / \partial \hat{\theta}$. This effect can be so strong that inequality (19) holds.

For $\delta$ very large, however, $J$ becomes so small that only a negligible fraction of the population receives confirmatory messages. The weighted average, $J \partial \hat{x}_{I} / \partial \hat{\theta}+(1-$ $J) \partial \hat{x}_{U} / \partial \hat{\theta}$, would dip below $\partial \hat{x}_{p s} / \partial \hat{\theta}$ again.

Proposition 5 also says that the relative precision of private information has to be reasonably high for communication to take a strong effect. When the noise is smaller in private information, citizens' private signals will be on average closer to the true $\theta$. A confirmatory message will be stronger evidence for the true strength being close to what the rumor says. Therefore, recipients of confirmatory messages will be more responsive to the rumor, making the left-hand-side of (19) bigger. In the "public signal model," on the other hand, a higher precision of the private signals means that citizens will rely more on their private signal and be less responsive to the rumor, making the right-hand-side of (19) smaller.

Our analysis of rumors suggests that, for outcomes of collective actions, it matters little whether rumors reflect the truth or have no basis in fact. What matters is that 
rumors create public topics that people can talk about. By communication, people learn from what others believe regarding the rumor and can better coordinate their actions. That explains why some false rumors could mobilize citizens very effectively when collective actions take place.

\subsection{Prior Belief and Sentiment}

Similar to Proposition 5, we can also establish that the effect of communication is smaller when $\alpha$ is closer to 1 or 0 , and is bigger when it takes intermediate values. If citizens have strong priors, their peers' assessments about the rumor have little effect. In contrast, if citizens are less certain about the source of rumors, information exchange among citizens has a stronger effect on the equilibrium.

The effect of changing $\alpha$ can be decomposed into three mechanisms. First, when the prior belief $\alpha$ is higher, the posterior belief $w$ that the rumor is informative is higher. Second, as a result, the fraction of citizens with $w \geq \delta$ is also higher. That is, $J$ increases when $w$ increases. Third, the counteracting effect is that, when the message sending interval widens and a larger fraction of citizens send confirmatory messages to their peers, recipients become less responsive.

When $\alpha$ is sufficiently small and becomes slightly larger, the first two effects dominate the third such that the effect of communication becomes larger. However, when $\alpha$ is large enough, both $w$ and $J$ are close to 1 and the increments to both are very small when $\alpha$ increases further. Therefore, the third effect dominates and recipients of confirmatory messages do not update their beliefs that much. That is, the effect of communication decreases as $\alpha$ increases beyond a certain point.

Similar analysis can be applied to the effects of changes in sentiment $s$. Consider the case where $s$ increases from "neutral sentiment" $s=z^{\prime}$ to infinity. ${ }^{25}$ If $s$ increases from the neutral value, citizens believe that a rumor against the regime is more likely to have come from an informative source. The aforementioned three mechanisms are still at work: both $w$ and $J$ increase and citizens are more reactive to a negative rumor but the resulting wider message sending interval implies that recipients of confirmatory messages become less responsive. The first two effects dominate the third if the sentiment is close to neutral. If $s$ is sufficiently large, both $w$ and $J$ are close to 1 . Since almost everybody thinks that a negative rumor is informative and says so to her peers, information exchange becomes less useful for citizens. In sum, the effect of communication also responds to $s$ in a non-monotone fashion.

\footnotetext{
${ }^{25}$ The sentiment $s=z^{\prime}$ is considered "neutral," since the equilibrium regime survival threshold is symmetric in $s$ about the point $s=z^{\prime}$.
} 


\section{Discussion}

\subsection{The Topic of Conversations Matters}

In the baseline model, we have assumed that communication takes the form of exchanging coarse (binary) signals about the informativeness of the rumor. In this subsection, we demonstrate that information coarsening is not a crucial assumption. It is the exchange of views on the rumor's informativeness that drives our results. This mechanism is qualitatively different from the case where the content of conversation is unrelated to the rumor. To stress this point, we explore two alternative communication protocols, which allow citizens to exchange the exact value of $w\left(z, x_{i}\right)$ or the exact value of $x_{i}$, respectively. We then discuss the case where both protocols are allowed.

Exchange $w\left(z, x_{i}\right)$. Suppose citizens can tell each other the value of $w$ (instead of just whether $w$ is greater than or less than $\delta$ ). Citizen $i$ updates her belief in a Bayesian fashion when she receives the message $w_{k}=w\left(z, x_{k}\right)$ from her peer $k$ :

$$
\operatorname{Pr}\left[\theta<\hat{\theta} \mid z, x_{i}, w_{k}\right]=\frac{\int_{-\infty}^{\hat{\theta}} j\left(t, z, w_{k}\right) p\left(t \mid z, x_{i}\right) \mathrm{d} t}{\int_{-\infty}^{\infty} j\left(t, z, w_{k}\right) p\left(t \mid z, x_{i}\right) \mathrm{d} t}
$$

where

$$
j\left(t, z, w_{k}\right)=\frac{1}{\sigma_{x}} \phi\left(\frac{x^{l}-t}{\sigma_{x}}\right)+\frac{1}{\sigma_{x}} \phi\left(\frac{x^{r}-t}{\sigma_{x}}\right),
$$

with $x^{l}$ and $x^{r}$ being the two values of $x_{k}$ that solves the equation $w\left(z, x_{k}\right)=w_{k}$.

The function $j\left(t, z, w_{k}\right)$ reaches a local maximum at $t=z$ if $w_{k}$ is high ( $x^{l}$ and $x^{r}$ are near $z$ ), or a local minimum at $t=z$ if $w_{k}$ is low ( $x^{l}$ and $x^{r}$ are far from $z$ ). Moreover, at $z=z^{\prime}$, there exists a $\hat{w}$ such that the ratio, $j\left(\theta=z^{\prime}, z^{\prime}, w_{k}\right) / \int_{-\infty}^{\infty} j\left(t, z^{\prime}, w_{k}\right) p\left(t \mid z^{\prime}, x^{\prime}\right) \mathrm{d} t$, is greater than 1 if $w_{k}>\hat{w}$. Therefore, citizens who receive a message $w_{k}>\hat{w}$ in this model are similar to citizens who receive a message $y=1$ in the baseline model. Similarly, the ratio $j\left(\theta=z^{\prime}, z^{\prime}, w_{k}\right) / \int_{-\infty}^{\infty} j\left(t, z^{\prime}, w_{k}\right) p\left(t \mid z^{\prime}, x^{\prime}\right) \mathrm{d} t$ is less than 1 if $w_{k}<\hat{w}$. Therefore, citizens who receive a message $w_{k}<\hat{w}$ in this model are similar to citizens who receive a message $y=0$ in the baseline model.

Intuitively, when citizen $k$ conveys a high assessment that the rumor is likely to be informative, i.e., $w_{k}>\hat{w}$, her peer $i$ assigns a higher probability weight (density) to states close to $z$. Similarly, when citizen $k$ does not believe the rumor is sufficiently informative, i.e., $w_{k}<\hat{w}$, citizen $i$ assigns lower weight to states close to $z$ and higher weight to states far away from $z$. Recall this mechanism of probability re-weighting is precisely the key that drives our results in the baseline case. Therefore, the model of exchanging the exact probability assessments is not qualitatively different from our 
model of exchanging coarse information.

Exchange $x_{i}$. Now we turn to the case where citizens directly exchange their private information. To be more general, we assume that the communication process is noisy: each citizen receives her peer's private signal with an additive noise. That is, the message $y_{i}$ received by the citizen $i$ from her peer $k$ is given by $y_{i}=x_{k}+\xi_{k}$, where the noise $\xi_{k} \sim \mathcal{N}\left(0, \sigma_{\tilde{\xi}}^{2}\right)$ is independent of $x_{k}$ and independently distributed across $k$. After communication, each citizen possesses an information set which consists of the rumor $z$ and two private signals $x_{i}$ and $y_{i}$. This information set is equivalent to $\left\{z, v_{i}\right\}$, where $v_{i}$ is a private signal with higher precision than $x_{i}$. That is,

$$
\begin{aligned}
v_{i} & \equiv \frac{\sigma_{x}^{2}+\sigma_{\xi}^{2}}{2 \sigma_{x}^{2}+\sigma_{\xi}^{2}} x_{i}+\frac{\sigma_{x}^{2}}{2 \sigma_{x}^{2}+\sigma_{\xi}^{2}} y_{i}, \\
\sigma_{v}^{2} & \equiv \frac{\sigma_{x}^{2}+\sigma_{\xi}^{2}}{2 \sigma_{x}^{2}+\sigma_{\xi}^{2}} \sigma_{x}^{2}<\sigma_{x}^{2} .
\end{aligned}
$$

In other words, this setting is observationally equivalent to the "mute model," with private signals of higher quality. An increase in the precision of private information causes citizens to be less responsive to the rumor in the "mute model."

The contrast between these two alternative models highlights that the topic of conversations matters: when citizens talk about what they privately know, they put less emphasis on public information; when they exchange views on the public signal that they commonly observe, they rely more on the public information.

Exchange both. Our analysis of communication is not affected if we allow citizens to exchange their private signal, as long as conversation about the rumor is also allowed. Consider a hybrid model by allowing citizen $i$ to exchange private information with a random peer $k$ and to exchange views on the informativeness of the rumor with another random peer $k^{\prime}$. As the previous analysis suggests, the exchange of private information only improves its precision.

Because our analysis holds true for any precision of private information, changing its precision would not affect our results. One might conjecture that our results will be undermined once we allow for private-information exchange, but this is not necessarily the case either. In fact, Proposition 5 implies that more precise private information (higher $\beta$ ) can strengthen the effect of the rumor under certain conditions.

\subsection{Strategic Communication}

There are two key features of communication protocol in the benchmark model that drive our main results regarding the effect of rumors. First, citizens follow an "inter- 
val" message sending rule, i.e., they send confirmatory messages when their private signals are within a certain interval. Second, this interval is not fixed. Instead, it is anchored by the rumor: the interval is symmetric around and shifts with the rumor's realization. The implication is that people confirm the veracity of the rumor when what they know is sufficiently close to it. We have seen how these two features contribute to making communication a potent mechanism to coordinate collective action.

Although non-strategic communication can be a realistic description of many conversations between acquaintances, especially when each individual has a negligible effect on the aggregate outcome, there are situations when strategic concerns also play a role. In times of turmoil, a citizen may not know who is a friend or an enemy, and she may have to be careful about what she tells her peer. In this subsection, we provide a model of strategic communication that captures some of these concerns. The resulting equilibrium communication rule is not mechanical as in the benchmark model, but still delivers the two key features of the benchmark communication protocol that drive our main results.

Assume that citizens are randomly paired up and play a communication game. Each citizen can choose to say "Yes, I believe it," or "No, I do not believe it." In contrast to the costless message sending setup in the benchmark model, citizens may incur two types of cost. First, if one's message is subsequently contradicted by the facts, the sender may face penalties for spreading false rumors or for instilling skepticism toward legitimate news. This is especially relevant in autocratic regimes where the authority tends to punish people who express sympathetic views on unverified rumors. Alternatively, the sender may also incur a psychological cost when she realizes that she had misled her peer. We let $c_{1}$ be the expected cost to a citizen when she says "yes" but the rumor turns out to be uninformative, and $c_{0}$ be the expected cost when she says "no" but the rumor turns out to be informative. Second, one does not fully know who one is talking to in a casual conversation. If a sender's assessment is at odds with that of her peer's, then she may face a chance that her peer may turn her in to the regime or to the rebels, so that she is singled out and punished. ${ }^{26}$ Thus, in sending a message to her peer, a citizen's cost depends on what the other citizen's message is. We let $d_{1}$ be the expected cost to a citizen if she says "yes" but her peer says "no," and let $d_{0}$ be the expected cost if she says "no" but her peer says "yes." The cost is normalized to 0 if both citizens express the same assessment. The cost matrix to player $i$ is summarized in Table 1 , where $\mathbb{1}\{\cdot\}$ is the indicator function. ${ }^{27}$

A message sending rule is a set $S$ such that a player chooses to express confirmatory

\footnotetext{
${ }^{26}$ When they have different assessments on the same rumor, one of them can be considered as being unfriendly to the regime. There is a chance that the other citizen betrays her and turns her in to the regime. Moreover, a citizen who expresses assessments that are sympathetic to the regime can also be
} 
Table 1. Player i's cost matrix.

\begin{tabular}{cccc}
\hline & \multicolumn{2}{c}{ Player $k$} \\
& "yes" & "no" \\
\hline Player $i$ & "yes" & $\mathbb{1}\{z \sim U\} \cdot c_{1}$ & $\mathbb{1}\{z \sim U\} \cdot c_{1}+d_{1}$ \\
& "no" & $\mathbb{1}\{z \sim I\} \cdot c_{0}+d_{0}$ & $\mathbb{1}\{z \sim I\} \cdot c_{0}$ \\
\hline
\end{tabular}

view if and only if her private information is in $S$. Given that player $i$ expects her peer player $k$ to adopt the message sending rule $S$, her assessment that player $k$ will send a confirmatory message is

$$
q\left(S ; z, x_{i}\right) \equiv w\left(z, x_{i}\right) \operatorname{Pr}\left[x_{k} \in S \mid z, x_{i}, z \sim I\right]+\left(1-w\left(z, x_{i}\right)\right) \operatorname{Pr}\left[x_{k} \in S \mid z, x_{i}, z \sim U\right] .
$$

Using the cost matrix in Table 1, player $i$ chooses to say "yes" if and only if

$$
q\left(S ; z, x_{i}\right) \geq \frac{d_{1}+c_{1}-w\left(z, x_{i}\right)\left(c_{0}+c_{1}\right)}{d_{0}+d_{1}} .
$$

The equilibrium message sending rule $S^{*}$ is a fixed point: given citizens follow the message sending rule $S^{*}$, inequality (20) holds if and only if $x_{i} \in S^{*}$.

Proposition 6. If $c_{1}>d_{0}>c_{1}-w(z, z)\left(c_{0}+c_{1}\right)$, there exists an interval equilibrium message sending rule $S^{*}$ and it is symmetric about the rumor $z$.

When $d_{0} \geq c_{1}$, it is an equilibrium that everybody endorses the rumor. If citizen $i$ expects that her peer will say "yes" and the cost $c_{1}$ of being punished for endorsing a false rumor is smaller relative to the cost $d_{0}$ of disagreeing with one's peer and being turned in to the regime or rebels, citizen $i$ chooses to say "yes" as well, and no meaningful communication occurs. On the other hand, when $d_{0} \leq c_{1}-w(z, z)\left(c_{0}+c_{1}\right)$, even the most "confident" citizen whose private information is confirmed by the rumor (i.e., $x_{i}=z$ ) finds it costly to endorse its veracity. In that case, no citizen would say "yes" even if she expects her peer to say "yes."

If $d_{0}$ is neither too high nor too low, (i.e., it satisfies the restriction stated in Proposition 6), then it can support an equilibrium in which citizens who find the rumor sufficiently plausible would choose to say that they believe the rumor. To see this,

turned in by her peer and punished by the rebels for betraying the cause of the revolution.

${ }^{27}$ Note that the cost parameters $c_{l}$ and $d_{l}$ can be functions of $z$. But it does not affect the equilibrium results, since citizens play this game after they observe the rumor. 
suppose that the message sending rule takes the form of an interval: $S=[\underline{x}, \bar{x}]$. Then,

$$
\begin{aligned}
q\left(S ; z, x_{i}\right)=w & \left(z, x_{i}\right)\left(\Phi\left(\frac{\bar{x}-\beta x_{i}-(1-\beta) z}{\sqrt{\beta+1} \sigma_{x}}\right)-\Phi\left(\frac{\underline{x}-\beta x_{i}-(1-\beta) z}{\sqrt{\beta+1} \sigma_{x}}\right)\right) \\
& +\left(1-w\left(z, x_{i}\right)\right)\left(\Phi\left(\frac{\bar{x}-x_{i}}{\sqrt{2} \sigma_{x}}\right)-\Phi\left(\frac{\underline{x}-x_{i}}{\sqrt{2} \sigma_{x}}\right)\right)
\end{aligned}
$$

If $S$ is symmetric about $z, q\left(S ; z, x_{i}\right)$ increases in $x_{i}$ from zero, peaks at $x_{i}=z$ and then decreases towards zero. Intuitively, when $x_{i}$ is extreme, player $i$ tends to believe that player $k^{\prime}$ s private signal is also very extreme and the probability that $x_{k}$ falls in any intermediate interval $S$ is low. Thus the left-hand-side of inequality (20) is humpshaped in $x_{i}$. Because of the property of $w\left(z, x_{i}\right)$, the right-hand-side of (20) is inverted hump-shaped in $x_{i}$. Proposition 6 shows that there exists an interval $[\underline{x}, \bar{x}]$ such that the left-hand-side exceeds the right-hand-side if and only if $x_{i}$ belongs to this interval. Further, this interval must contain $z$ and is symmetric about it. For any symmetric decision rule $S$, both $q(S ; z, \cdot)$ and $w(z, \cdot)$ are symmetric about the rumor $z$. Given the symmetry of both sides of (20), the equilibrium message sending rule $S^{*}(z)$ is symmetric as well.

Depending on the cost parameters, the endogenous decision interval $S^{*}(z)$ may be larger or smaller than that specified in the benchmark case with exogenous plausibility threshold $\delta$. Instead of reporting whether they believe a rumor mechanically, citizens choose their messages depending on expected costs and on what they believe their peers will report. However, this equilibrium communication rule shares the same key features as the exogenous communication rule in the benchmark case. In terms of the effect of communication on equilibrium in the attack stage of the game, the two alternative communication protocols deliver the same qualitative results.

\subsection{Robustness}

Our baseline specification on communication is the simplest possible one that allows citizens to exchange views on the informativeness of the rumor. When we relax various simplifying assumptions, our main results still hold. We discuss three examples.

Finer messages. First, we investigate the case where citizens can exchange finer messages, rather than simply $y=1$ or 0 . Specifically, if citizens decide to send their peers contradictory messages $y=0$, we allow them to justify and explain why they do not believe the rumor is informative. When $x_{k}<\underline{x}(z)$, citizen $k$ can send her peer a message $y=0 L$, interpreted to mean "the rumor is not informative because it indicates that the regime is too much stronger than I believe;" she sends a message $y=0 R$ when $x_{k}>\bar{x}(z)$; and she sends $y=1$ when $x_{k} \in[\underline{x}(z), \bar{x}(z)]$. 
In the baseline model, the key result that rumors can be even more potent than trustworthy news is driven by the fact that the effect of communication can dominate skepticism when a confirmatory message is received and by the fact that the fraction of population who receive those messages can be sufficiently large. The same driving force can also dominate in this specification and lead to a similar result.

Meeting with like-minded citizens. Second, in our benchmark model, we assume that citizens meet in a completely random manner. However, one may argue that in reality, people are more likely to meet someone who shares similar beliefs. To capture the spirit of such a semi-random meeting technology, we assume that citizens split into two groups after they hear the rumor: those whose private information is lower than the value of $z$ form a group $L$ and the rest form a group $R$. Then citizens are randomly matched in pairs in each group. Citizen $k$ from $L$ sends her peer a message $y=0$ when $x_{k}<\underline{x}(z)$; or $y=1$, when $\underline{x}(z)<x_{k}<z$. Similarly, citizen $k^{\prime}$ from $R$ sends her peer $y=0$ when $x_{k^{\prime}}>\bar{x}(z)$; or $y=1$ when $z<x_{k^{\prime}}<\bar{x}(z)$.

This setup is quite similar to the specification of exchanging finer messages discussed above. The difference is that the group of citizens who receive $y=1$ in that case splits into two in this case. However, recipients of confirmatory messages in both groups consider those messages as evidence for $\theta$ being close to $z$. The same logic of the "finer messages" case continues to apply here and rumors can still be more potent than trustworthy news in this case.

Meeting with more than one citizen. Third, we can also extend our model to the case where citizens meet up and communicate in a group. Specifically, $N$ citizens are randomly grouped together in the communication stage and then each of them sends one message to and receives $N-1$ messages from his $N-1$ group members simultaneously. Naturally, there are $N$ types of citizens after they communicate, depending on the number of confirmatory messages received, $n$, where $n \in\{0,1, \ldots, N-1\}$. It turns out that the posterior densities of citizens that receive a larger number of confirmatory messages are more concentrated around the rumor and therefore become more responsive to it. It is also similar to the benchmark case in that the fraction of citizens who receive a high number of confirmatory messages is larger if the rumor happens to be closer to the true fundamental. Therefore, our key results can still extend. ${ }^{28}$

\footnotetext{
${ }^{28} \mathrm{~A}$ formal characterization and results from the case with $N=3$ are available upon request. Note that the case where infinitely many citizens can meet up and exchange information is different. If citizens receive infinitely many messages, by the law of large numbers, they can back out the fundamental $\theta$ given the fraction of confirmatory messages they receive and the message sending rule.
} 


\subsection{Censorship: The Power of Silence}

As rumors can be powerful in mobilizing citizens to revolt, autocratic governments may want to block rumors against them and to stop citizens from talking about these rumors. However, does it always help the regime to survive if censorship is adopted to screen out all negative information? When citizens hear no rumor about the regime, silence itself becomes a public signal about the regime strength, and it is not obvious that censorship necessarily increases the chances of regime survival.

Assume that the regime blocks any rumor $z$ if $z<K$ and citizens are aware of this censorship rule. When $z$ is observable, the equilibrium is the same as in our "communication model." When citizens hear no rumor, they understand that the authority has blocked the rumor and $z<K$. Taking $\theta_{c}$ as the threshold for regime survival, citizens calculate the expected payoff of revolt in a Bayesian fashion,

$$
\operatorname{Pr}\left[\theta \leq \theta_{c} \mid z<K, x_{i}\right]=\frac{\int_{-\infty}^{\theta_{c}}\left[\alpha \Phi\left(\frac{K-t}{\sigma_{z}}\right)+(1-\alpha) \Phi\left(\frac{K-s}{\sigma_{U}}\right)\right] \frac{1}{\sigma_{x}} \phi\left(\frac{t-x_{i}}{\sigma_{x}}\right) \mathrm{d} t}{\int_{-\infty}^{\infty}\left[\alpha \Phi\left(\frac{K-t}{\sigma_{z}}\right)+(1-\alpha) \Phi\left(\frac{K-s}{\sigma_{U}}\right)\right] \frac{1}{\sigma_{x}} \phi\left(\frac{t-x_{i}}{\sigma_{x}}\right) \mathrm{d} t} .
$$

Now we examine the effect of censorship on regime survival, when the regime blocks any negative rumors against it, i.e., $K=z^{\prime}$. The "censorship model" is a variant of the "mute model" when rumors are blocked, since citizens cannot communicate when there is no rumor to talk about. In a sense, citizens pool the effects of negative rumors, both more and less dangerous ones, and use an average to make a decision, given that they cannot observe the censored rumor $z$. Therefore, censorship does help the regime to survive when the blocked rumors happen to be the most dangerous ones (i.e., when $\theta^{*}(z)$ is around the peak value), but hurts the regime when they are extreme or close to neutral. ${ }^{29}$ See Figure 6 for illustration.

\section{Conclusion}

Social interaction is an important source of information for individuals, especially when the coordination motive is important. Our paper highlights the significance of this channel and contributes to this interesting but under-explored topic.

It is not news that revolutions in history are often intertwined with rumors. However, what strikes us is why some rumors, which often turned out to be false later,

\footnotetext{
${ }^{29}$ Our analysis specifically highlights the effects of censoring rumors on the survival of the regime. More detailed analysis can be obtained upon request. The optimal censorship strategy is not the focus of this paper and it is systematically characterized in a more general setup, developed by Shadmehr and Bernhardt (2013). They study state censorship in a model with a ruler, a representative citizen and media, where a similar mechanism also arises, that "no news" becomes "bad news."
} 


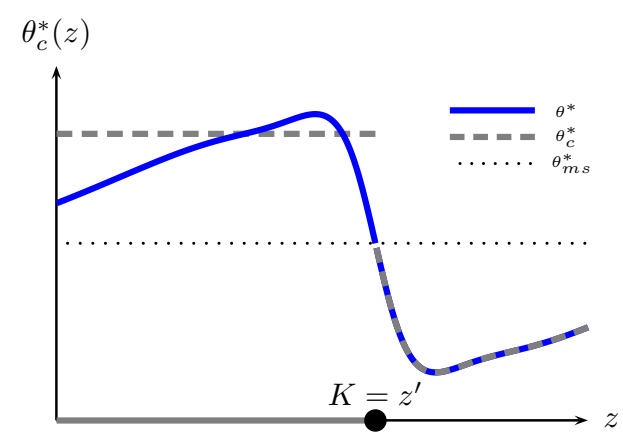

Figure 6. The dashed line shows the equilibrium survival threshold in the "censorship model," whereas the solid line represents that in the "communication model." Censorship can hurt the regime by raising the survival threshold above that in the "communication model."

could be so effective for mobilization, while others were simply ignored. We offer an analysis of this phenomenon by focusing on two key aspects of rumors: that they may or may not be true, and that people talk about them. In this model, individuals' skepticism toward rumors arises as a rational response instead of a behavioral assumption. Moreover, we explicate a novel mechanism where the effect of citizens' skepticism can be undone or reinforced by communication among themselves.

To the best of our knowledge, our model is the first attempt to explicitly investigate the role of rumors in a regime change game. Our theory is interpreted in the context of political revolution, but it can also be extended to model rumors in bank runs, financial crises and currency attacks. We have not, however, addressed questions about how rumors originate or how they spread. Although we explore a number of communication protocols in this paper, our analysis is confined to decentralized communication with pairwise matching. The role played by social networks, mass media, and modern communication technologies in promulgating or abating rumors remains to be studied. 


\section{Appendix}

Proof of Proposition 1. Part (a). Combine the indifference condition and the critical mass conditions in the "mute model," we get

$$
\theta_{m}^{*}=(1-c)+\left[\Phi\left(\frac{\theta_{m}^{*}-\left(\beta x_{m}^{*}+(1-\beta) z\right)}{\sqrt{\beta} \sigma_{x}}\right)-c\right] \cdot \frac{w\left(z, x_{m}^{*}\right)}{1-w\left(z, x_{m}^{*}\right)}
$$

For any finite $x_{m}^{*}, \lim _{z \rightarrow \infty} w\left(z, x_{m}^{*}\right)=0$. Therefore,

$$
\lim _{z \rightarrow \infty} \theta_{m}^{*}(z)=1-c=\theta_{m s}^{*}
$$

As $z$ goes to infinity, $x_{m}^{*}(z)$ must remain finite. To see why this is the case, suppose $z$ is sufficiently large and $x^{*}$ goes to negative infinity, the equation above implies that $\theta_{m}^{*}=$ $1-c$ and $A\left(\theta_{m}^{*} ; z\right)=0$. The critical mass condition is violated. Next suppose that $z$ is sufficiently large and $x^{*}$ goes to infinity. The equation above implies that $\theta_{m}^{*}<1-c$, but the fact that $x^{*}$ is sufficiently large implies that $A\left(\theta_{m}^{*} ; z\right)=1$. The critical mass condition is again violated. A similar argument establishes that $\lim _{z \rightarrow-\infty} \theta_{m}^{*}(z)=\theta_{m s}^{*}$.

Part (b). We first show that if $z<z^{\prime}$, then $\theta_{m}^{*}(z)<\theta_{p s}^{*}(z)$. From the critical mass condition in the "mute model," we have $\Phi\left(\sigma_{x}^{-1}\left(\theta_{m}^{*}-x_{m}^{*}\right)\right)=1-\theta_{m}^{*}$. Therefore, the indifference condition in the "mute model" can be written as:

$$
c=w\left(z, x_{m}^{*}\right) \Phi\left(\frac{\theta_{m}^{*}-\left(\beta x_{m}^{*}+(1-\beta) z\right)}{\sqrt{\beta} \sigma_{x}}\right)+\left(1-w\left(z, x_{m}^{*}\right)\right)\left(1-\theta_{m}^{*}\right) .
$$

From the indifference condition of the "public signal model" and from the fact that $1-\theta_{m s}^{*}=c$, we also have

$$
c=w\left(z, x_{m}^{*}\right) \Phi\left(\frac{\theta_{p s}^{*}-\left(\beta x_{p s}^{*}+(1-\beta) z\right)}{\sqrt{\beta} \sigma_{x}}\right)+\left(1-w\left(z, x_{m}^{*}\right)\right)\left(1-\theta_{m s}^{*}\right) .
$$

These two equations, together with the fact that $\theta_{p s}^{*}>\theta_{m s}^{*}$ when $z<z^{\prime}$, imply

$$
\begin{array}{r}
w\left(z, x_{m}^{*}\right) \Phi\left(\frac{g\left(\theta_{m}^{*}\right)-(1-\beta) z}{\sqrt{\beta} \sigma_{x}}\right)+\left(1-w\left(z, x_{m}^{*}\right)\right)\left(1-\theta_{m}^{*}\right) \\
>w\left(z, x_{m}^{*}\right) \Phi\left(\frac{g\left(\theta_{p s}^{*}\right)-(1-\beta) z}{\sqrt{\beta} \sigma_{x}}\right)+\left(1-w\left(z, x_{m}^{*}\right)\right)\left(1-\theta_{p s}^{*}\right),
\end{array}
$$

where

$$
g\left(\theta^{*}\right) \equiv \theta^{*}-\beta x^{*}=\theta^{*}-\beta\left(\theta^{*}+\sigma_{x} \Phi^{-1}\left(\theta^{*}\right)\right) .
$$

To show $\theta_{m}^{*}<\theta_{p s}^{*}$ from the above inequality, it suffices to show that $\mathrm{d} g\left(\theta^{*}\right) / \mathrm{d} \theta^{*} \leq 0$. 
We have

$$
\frac{\mathrm{d} g\left(\theta^{*}\right)}{\mathrm{d} \theta^{*}}=1-\beta-\frac{\beta \sigma_{x}}{\phi\left(\Phi^{-1}\left(\theta^{*}\right)\right)} \leq 1-\beta-\beta \sigma_{x} \sqrt{2 \pi},
$$

which is negative by assumption (1). Hence $\theta_{m}^{*}<\theta_{p s}^{*}$ when $z<z^{\prime}$.

Next, we show that $\theta_{m}^{*}(z)>\theta_{m s}^{*}$ if $z<z^{\prime}$. Suppose this is not true. Then $\Phi\left(\sigma_{x}^{-1}\left(\theta_{m}^{*}-\right.\right.$ $\left.\left.x_{m}^{*}\right)\right)=1-\theta_{m}^{*} \geq 1-\theta_{m s}^{*}=c$, which implies

$$
\Phi\left(\frac{\theta_{m}^{*}-\left(\beta x_{m}^{*}+(1-\beta) z\right)}{\sqrt{\beta} \sigma_{x}}\right) \leq c .
$$

Moreover, the fact that $\left(\theta_{m s}^{*}, x_{m s}^{*}\right)$ satisfies the indifference condition of the "public signal model" at $z=z^{\prime}$ implies

$$
\Phi\left(\frac{\theta_{m s}^{*}-\left(\beta x_{m s}^{*}+(1-\beta) z^{\prime}\right)}{\sqrt{\beta} \sigma_{x}}\right)=c .
$$

These two conditions can be combined to give

$$
g\left(\theta_{m}^{*}\right)-g\left(\theta_{m s}^{*}\right) \leq(1-\beta)\left(z-z^{\prime}\right)<0 .
$$

Since $\mathrm{d} g\left(\theta^{*}\right) / \mathrm{d} \theta^{*}<0$, this inequality implies $\theta_{m}^{*}>\theta_{m s}^{*}$, a contradiction. Thus, when $z<z^{\prime}$, we must have $\theta_{m}^{*}(z) \in\left(\theta_{m s}^{*}, \theta_{p s}^{*}(z)\right)$. When $z \geq z^{\prime}$, the proof is symmetric.

Part (c). The following is to show that (i) $\theta_{m}^{*}(z)$ is increasing and then decreasing for $z \in\left(-\infty, z^{\prime}\right)$; and (ii) $\theta_{m}^{*}(z)$ is decreasing and then increasing for $z \in\left(z^{\prime}, \infty\right)$. Fix a $z_{0} \in\left(-\infty, z^{\prime}\right)$.

Define

$$
f(z) \equiv P\left(\theta_{m}^{*}\left(z_{0}\right) \mid z, x_{m}^{*}\left(z_{0}\right)\right) \text {. }
$$

We show that $f$ is single-peaked in $z$ for $z \in\left(-\infty, z^{\prime}\right)$. It suffices to verify that $\mathrm{d} f(z) / \mathrm{d} z=0$ implies $\mathrm{d}^{2} f(z) / \mathrm{d} z^{2}<0$. To simplify the notation, we use the subscript $I$ or $U$ to denote the posterior distribution (or density) when $z$ is known to be informative or uninformative, respectively. We have

$$
\begin{aligned}
\frac{\mathrm{d} f(z)}{\mathrm{d} z} & =\left(\Phi_{I}-\Phi_{U}\right) \frac{\partial w}{\partial z}-w \frac{\partial \Phi_{I}}{\partial z} \\
& =\left[(1-w)\left(\frac{z-s}{\sigma_{U}^{2}}+\frac{x_{m}^{*}\left(z_{0}\right)-z}{\sigma_{I}^{2}}\right)\left(1-\frac{\Phi_{U}}{\Phi_{I}}\right)-\frac{1-\beta}{\sqrt{\beta} \sigma_{x}} \frac{\phi_{I}}{\Phi_{I}}\right] w \Phi_{I} .
\end{aligned}
$$


When $\mathrm{d} f(z) / \mathrm{d} z=0$, the second derivative is given by:

$$
\begin{aligned}
\frac{1}{w \Phi_{I}} \frac{\mathrm{d}^{2} f(z)}{\mathrm{d} z^{2}}=- & w(1-w)\left(\frac{z-s}{\sigma_{U}^{2}}+\frac{x_{m}^{*}\left(z_{0}\right)-z}{\sigma_{I}^{2}}\right)^{2}\left(1-\frac{\Phi_{U}}{\Phi_{I}}\right) \\
& +(1-w)\left(\frac{1}{\sigma_{U}^{2}}-\frac{1}{\sigma_{I}^{2}}\right)\left(1-\frac{\Phi_{U}}{\Phi_{I}}\right) \\
& -(1-w)\left(\frac{z-s}{\sigma_{U}^{2}}+\frac{x_{m}^{*}\left(z_{0}\right)-z}{\sigma_{I}^{2}}\right)\left(\frac{\partial\left(\Phi_{U} / \Phi_{I}\right)}{\partial z}\right) \\
& -\left(\frac{1-\beta}{\sigma_{x} \sqrt{\beta}}\right) \frac{\partial\left(\phi_{I} / \Phi_{I}\right)}{\partial z} .
\end{aligned}
$$

The first term is negative because $\Phi_{I}>\Phi_{U}$ for $z<z^{\prime}$. To see this, suppose the contrary is true. Then $\Phi_{I} \leq \Phi_{U}$ implies

$$
\theta_{m}^{*}\left(z_{0}\right)+\sqrt{\beta} x_{m}^{*}\left(z_{0}\right) \leq(1+\sqrt{\beta}) z
$$

But for $z_{0} \leq z^{\prime}$ the left-hand side is greater than $\theta_{m s}^{*}+\sqrt{\beta} x_{m s}^{*}$, which is equal to $(1+$ $\sqrt{\beta}) z^{\prime}$, a contradiction. The second term is negative because $\sigma_{U}^{2}>\sigma_{I}^{2}$ from parameter restriction (2). The third term is negative because $(z-s) / \sigma_{U}^{2}+\left(x_{m}^{*}\left(z_{0}\right)-z\right) / \sigma_{I}^{2}>0$ whenever $\mathrm{d} f(z) / \mathrm{d} z=0$ and because $\Phi_{U} / \Phi_{I}$ is increasing in $z$. The fourth term is also negative because the function $\phi_{I} / \Phi_{I}$ is increasing in $z$.

The single-peakedness of $f(z)$ for $z \in\left(-\infty, z^{\prime}\right)$ implies that in this range there can be at most one $z_{1} \neq z_{0}$ such that $\theta_{m}^{*}\left(z_{1}\right)=\theta_{m}^{*}\left(z_{0}\right)$. Suppose otherwise. Let $z_{1} \neq z_{2} \neq z_{0}$ be such that $\theta_{m}^{*}\left(z_{1}\right)=\theta_{m}^{*}\left(z_{2}\right)=\theta_{m}^{*}\left(z_{0}\right)$. By the critical mass condition, this implies $x_{m}^{*}\left(z_{1}\right)=x_{m}^{*}\left(z_{2}\right)=x_{m}^{*}\left(z_{0}\right)$. Since $\left(\theta_{m}^{*}\left(z_{0}\right), x_{m}^{*}\left(z_{0}\right)\right)$ satisfies the equilibrium conditions for $z \in\left\{z_{1}, z_{2}, z_{0}\right\}$, the equation $f(z)=c$ has at least three solutions, which contradicts the single-peakedness of $f$.

In parts (a) and (b) of the proposition, we have already established that $\theta_{m}^{*}(z)$ is higher than $\theta_{m s}^{*}$ for $z \in\left(-\infty, z^{\prime}\right)$, and approaches it when $z$ goes to minus infinity or to $z^{\prime}$. Thus, $\theta_{m}^{*}(z)$ must be increasing for $z$ sufficiently negative, and decreasing for $z$ sufficiently close to $z^{\prime}$. Together with the fact that for any $z_{0}$ in this range, there can be at most one $z_{1}$ such that $\theta_{m}^{*}\left(z_{0}\right)=\theta_{m}^{*}\left(z_{1}\right)$, this implies that $\theta_{m}^{*}(z)$ must be increasing then decreasing in this range.

For the case $z \in\left(z^{\prime}, \infty\right)$, write:

$$
\frac{\mathrm{d} f(z)}{\mathrm{d} z}=\left[(1-w)\left(\frac{z-s}{\sigma_{U}^{2}}+\frac{x_{m}^{*}\left(z_{0}\right)-z}{\sigma_{I}^{2}}\right)\left(\frac{1-\Phi_{U}}{1-\Phi_{I}}-1\right)-\frac{1-\beta}{\sqrt{\beta} \sigma_{x}} \frac{\phi_{I}}{1-\Phi_{I}}\right] w\left(1-\Phi_{I}\right) .
$$


We can show that the bracketed term is increasing when it is equal to zero, because $\Phi_{I}<\Phi_{U}$ when $z>z^{\prime}$ and because $\phi_{I} /\left(1-\Phi_{I}\right)$ is decreasing in $z$. Hence $f(z)$ must be decreasing then increasing in $z$ in this range. Following similar reasoning as in the earlier case, this implies that $\theta_{m}^{*}(z)$ is decreasing then increasing for $z \in\left(z^{\prime}, \infty\right)$.

Proof of Proposition 2. Part (a). We first show that there exists unique $\tilde{z}$ such that $x_{I}(\tilde{z})=x_{U}(\tilde{z})=x_{m}(\tilde{z})$, and then show that $\tilde{z}=z^{\prime}$ when $c=0.5$.

From Lemma 10 in the Technical Appendix, $x_{I}^{*}(z)>x_{U}^{*}(z)$ for $z$ sufficiently negative and $x_{I}^{*}(z)<x_{U}^{*}(z)$ for $z$ sufficiently large. Both $x_{I}^{*}(z)$ and $x_{U}^{*}(z)$ are continuous. Therefore there exists a $\tilde{z}$ such that $x_{I}^{*}(\tilde{z})=x_{U}^{*}(\tilde{z})$.

Let $\theta^{*}(\tilde{z})=\tilde{\theta}$ and $x_{I}^{*}(\tilde{z})=x_{U}^{*}(\tilde{z})=\tilde{x}$. We proceed to establish that $(\tilde{\theta}, \tilde{x})$ solves the "mute model" as well. To see this, we first note that $x_{I}^{*}=x_{U}^{*}$ implies that the critical mass condition (10) of the "communication model" reduces to its counterpart in the "mute model." Next, note that for any value of $\tilde{z}, \tilde{x}$ and $\tilde{\theta}$, we have

$$
P(\tilde{\theta} \mid \tilde{z}, \tilde{x})=\operatorname{Pr}\left[y_{i}=1 \mid \tilde{z}, \tilde{x}\right] P(\tilde{\theta} \mid \tilde{z}, \tilde{x}, 1)+\operatorname{Pr}\left[y_{i}=0 \mid \tilde{z}, \tilde{x}\right] P(\tilde{\theta} \mid \tilde{z}, \tilde{x}, 0)
$$

Thus, if $\tilde{z}, \tilde{x}$, and $\tilde{\theta}$ satisfy the indifference conditions $P(\tilde{\theta} \mid \tilde{z}, \tilde{x}, 1)=c$ and $P(\tilde{\theta} \mid \tilde{z}, \tilde{x}, 0)=$ $c$ in the "communication model," then they must satisfy the indifference condition $P(\tilde{\theta} \mid \tilde{z}, \tilde{x})=c$ in the "mute model" as well.

Let $\left(\theta^{\prime}, x^{\prime}\right)$ solve the "mute model" at $z=z^{\prime}$. To show $z^{\prime}=\tilde{z}$ when $c=0.5$, it suffices to show that $P\left(\theta^{\prime} \mid z^{\prime}, x^{\prime}, 1\right)=c$. That is because, given that $P\left(\theta^{\prime} \mid z^{\prime}, x^{\prime}\right)=c$, these two conditions would imply that $P\left(\theta^{\prime} \mid z^{\prime}, x^{\prime}, 0\right)=c$. Hence the indifference condition (9) for the "communication model" is satisfied. Given that $x_{I}^{*}\left(z^{\prime}\right)=x_{U}^{*}\left(z^{\prime}\right)=x^{\prime}$, the critical mass condition (10) holds as well.

To see why this condition holds, note that both $J\left(t, z^{\prime}\right)$ and $p\left(t \mid z^{\prime}, z^{\prime}\right)$ are symmetric about the point $t=z^{\prime}$, which gives

$$
\int_{-\infty}^{z^{\prime}} J\left(t, z^{\prime}\right) p\left(t \mid z^{\prime}, z^{\prime}\right) \mathrm{d} t=0.5 \int_{-\infty}^{\infty} J\left(t, z^{\prime}\right) p\left(t \mid z^{\prime}, z^{\prime}\right) \mathrm{d} t
$$

Hence, $P\left(z^{\prime} \mid z^{\prime}, z^{\prime}, 1\right)=0.5$. For $c=0.5, \theta^{\prime}=x^{\prime}=z^{\prime}$. Therefore, we have $P\left(\theta^{\prime} \mid z^{\prime}, x^{\prime}, 1\right)=$ c. This establishes that $\theta^{*}\left(z^{\prime}\right)=\theta_{m}^{*}\left(z^{\prime}\right)$ and $x_{I}^{*}\left(z^{\prime}\right)=x_{U}^{*}\left(z^{\prime}\right)=x_{m}^{*}\left(z^{\prime}\right)$.

Part (b). We show that $x_{I}^{*}(z)>x_{U}^{*}(z)$ for $z<z^{\prime}$. Suppose otherwise. Then, since $\lim _{z \rightarrow-\infty} x_{I}^{*}(z)>\lim _{z \rightarrow-\infty} x_{U}^{*}(z)$, there must exist some $z_{0}<z^{\prime}$ such that $x_{I}^{*}\left(z_{0}\right)=$ $x_{U}^{*}\left(z_{0}\right)$. According to Lemma 5 in the Technical Appendix, for any $z_{0}$, there exists a unique pair $\left(\hat{\theta}_{0}, x_{0}\right)$ such that $P\left(\hat{\theta}_{0} \mid z_{0}, x_{0}, 1\right)=P\left(\hat{\theta}_{0} \mid z_{0}, x_{0}, 0\right)=c$. It is easy to verify that such a pair is $\left(\hat{\theta}_{0}, x_{0}\right)=\left(z_{0}, z_{0}\right)$. However, such a pair does not satisfy the critical 
mass condition, because $A\left(z_{0} ; z_{0}, z_{0}, z_{0}\right) \neq z_{0}$, a contradiction. A symmetric argument shows that $x_{I}^{*}(z)<x_{U}^{*}(z)$ for $z>z^{\prime}$.

Proof of Proposition 3. We prove the second half of this proposition; the proof of the first part is analogous. We proceed by constructing a contradiction. Suppose $\theta^{*}(z) \leq$ $\theta_{m}^{*}(z)$ for $z$ sufficiently large. By Lemma 7 in the Technical Appendix, $0<\partial \hat{x}_{m} / \partial \hat{\theta}<1$ when $z$ is sufficiently large. This implies

$$
x_{m}^{*}\left(\theta_{m}^{*}(z)\right)-\theta_{m}^{*}(z) \leq x_{m}^{*}\left(\theta^{*}(z)\right)-\theta^{*}(z) .
$$

Since $\Phi$ is monotone, the above inequality together with the critical mass condition for the "mute model" implies:

$$
\theta_{m}^{*}(z)=\Phi\left(\frac{x_{m}^{*}\left(\theta_{m}^{*}(z)\right)-\theta_{m}^{*}(z)}{\sigma_{x}}\right) \leq \Phi\left(\frac{x_{m}^{*}\left(\theta^{*}(z)\right)-\theta^{*}(z)}{\sigma_{x}}\right)
$$

Lemma 8 in the Technical Appendix establishes that, for $z$ sufficiently large and for any $\hat{\theta}$,

$$
(1-J(\hat{\theta}, z)) \Phi\left(\frac{\hat{x}_{U}(\hat{\theta})-\hat{\theta}}{\sigma_{x}}\right)>\Phi\left(\frac{\hat{x}_{m}(\hat{\theta})-\hat{\theta}}{\sigma_{x}}\right),
$$

which implies that

$$
J(\hat{\theta}, z) \Phi\left(\frac{\hat{x}_{I}(\hat{\theta})-\hat{\theta}}{\sigma_{x}}\right)+(1-J(\hat{\theta}, z)) \Phi\left(\frac{\hat{x}_{U}(\hat{\theta})-\hat{\theta}}{\sigma_{x}}\right)>\Phi\left(\frac{\hat{x}_{m}(\hat{\theta})-\hat{\theta}}{\sigma_{x}}\right) .
$$

Evaluating both sides at $\hat{\theta}=\theta^{*}(z)$, it follows that

$$
\theta^{*}(z)>\Phi\left(\frac{x_{m}^{*}\left(\theta^{*}(z)\right)-\theta^{*}(z)}{\sigma_{x}}\right) \geq \theta_{m}^{*}(z)
$$

a contradiction.

Proof of Proposition 4. Recall that $\theta^{\prime}=\theta^{*}\left(z^{\prime}\right)$ and $x_{I}^{*}\left(z^{\prime}\right)=x_{U}^{*}\left(z^{\prime}\right)=x^{\prime}$ from the 
proof of Proposition 2. By Claim 2 in the proof of Lemma 2,

$$
\begin{aligned}
& J \frac{\partial \hat{x}_{I}}{\partial \hat{\theta}}+(1-J) \frac{\partial \hat{x}_{U}}{\partial \hat{\theta}} \\
= & -p\left(\theta^{\prime} \mid z^{\prime}, x^{\prime}\right)\left[J\left(\theta^{\prime}, z^{\prime}\right) \frac{1}{\int_{-\infty}^{\theta^{\prime}} \frac{J\left(t, z^{\prime}\right)}{J\left(\theta^{\prime}, z^{\prime}\right)} \frac{\partial p\left(t \mid z^{\prime}, x^{\prime}\right)}{\partial x} \mathrm{~d} t}+\left(1-J\left(\theta^{\prime}, z^{\prime}\right)\right) \frac{1}{\int_{-\infty}^{\theta^{\prime}} \frac{1-J\left(t, z^{\prime}\right)}{1-J\left(\theta^{\prime}, z^{\prime}\right)} \frac{\partial p\left(t \mid z^{\prime}, x^{\prime}\right)}{\partial x} \mathrm{~d} t}\right] \\
> & -p\left(\theta^{\prime} \mid z^{\prime}, x^{\prime}\right) \frac{1}{J\left(\theta^{\prime}, z^{\prime}\right) \int_{-\infty}^{\theta^{\prime}} \frac{J\left(t, z^{\prime}\right)}{J\left(\theta^{\prime}, z^{\prime}\right)} \frac{\partial p\left(t \mid z^{\prime}, x^{\prime}\right)}{\partial x} \mathrm{~d} t+\left(1-J\left(\theta^{\prime}, z^{\prime}\right)\right) \int_{-\infty}^{\theta^{\prime}} \frac{1-J\left(t, z^{\prime}\right)}{1-J\left(\theta^{\prime}, z^{\prime}\right)} \frac{\partial p\left(t \mid z^{\prime}, x^{\prime}\right)}{\partial x} \mathrm{~d} t} \\
= & \frac{-p\left(\theta \mid z^{\prime}, x^{\prime}\right)}{\int_{-\infty}^{\theta^{\prime}} \frac{\partial p\left(t \mid z^{\prime}, x^{\prime}\right)}{\partial x} \mathrm{~d} t} \\
= & \frac{\partial \hat{x}_{m}}{\partial \hat{\theta}}
\end{aligned}
$$

where the inequality follows from Jensen's inequality and the fact that the function $1 / t$ is concave for $t<0$. This establishes the first inequality of (18). Moreover, since

$$
P_{x}=-p+\frac{(1-\beta) w}{\sqrt{\beta} \sigma_{x}} \phi\left(\frac{\theta^{\prime}-X^{\prime}}{\sqrt{\beta} \sigma_{x}}\right)<0,
$$

we have $\partial \hat{x}_{m} / \partial \hat{\theta}=-p / P_{x}>1$. This establishes the second inequality of (18).

Inequality (17) follows from (18) and from Lemma 6 in the Technical Appendix, which shows that $\partial \hat{x} / \partial z=1-\partial \hat{x} / \partial \hat{\theta}$. Finally, using the fact that $\hat{x}_{I}\left(\theta^{\prime}, z^{\prime}\right)=\hat{x}_{U}\left(\theta^{\prime}, z^{\prime}\right)$, we can differentiate the critical mass condition (10) to obtain:

$$
\frac{\mathrm{d} \theta^{*}\left(z^{\prime}\right)}{\mathrm{d} z}=\frac{\frac{1}{\sigma_{x}} \phi(\cdot)\left(J \frac{\partial \hat{x}_{I}}{\partial z}+(1-J) \frac{\partial \hat{x}_{U}}{\partial z}\right)}{1-\frac{1}{\sigma_{x}} \phi(\cdot)\left(-1+J \frac{\partial \hat{x}_{I}}{\partial \hat{\theta}}+(1-J) \frac{\partial \hat{x}_{U}}{\partial \hat{\theta}}\right)} .
$$

Comparing this equation with its counterpart in the "mute model" (which is obtained by letting $J=1$ and replace $\partial \hat{x}_{I} / \partial z$ and $\partial \hat{x}_{I} / \partial \hat{\theta}$ with $\partial \hat{x}_{m} / \partial z$ and $\left.\partial \hat{x}_{m} / \partial \hat{\theta}\right)$, and using inequalities (17) and (18), we have:

$$
\frac{\mathrm{d} \theta^{*}\left(z^{\prime}\right)}{\mathrm{d} z}<\frac{\mathrm{d} \theta_{m}^{*}\left(z^{\prime}\right)}{\mathrm{d} z}<0
$$

Proposition 4 follows.

Proof of Proposition 5. We show that for $\beta$ larger than a threshold $\hat{\beta}$, there exists an interval $\left[\delta_{1}, \delta_{2}\right]$ such that, for any $\delta \in\left[\delta_{1}, \delta_{2}\right], \theta^{*}(z)>\theta_{p s}^{*}(z)$ if $z<z^{\prime}$. 
We write the difference between $\partial \hat{x}_{p s} / \partial \hat{\theta}$ and $\partial \hat{x}_{m} / \partial \hat{\theta}$ as:

$$
\frac{\partial \hat{x}_{p s}}{\partial \hat{\theta}}-\frac{\partial \hat{x}_{m}}{\partial \hat{\theta}}=\left(\frac{1-\beta}{\beta} \frac{1-w}{w \frac{1}{\sqrt{\beta}}+(1-w)}\right) \frac{\partial \hat{x}_{m}}{\partial \hat{\theta}} \equiv L_{p s} \frac{\partial \hat{x}_{m}}{\partial \hat{\theta}}
$$

and we write:

$$
\left(J \frac{\partial \hat{x}_{I}}{\partial \hat{\theta}}+(1-J) \frac{\partial \hat{x}_{U}}{\partial \hat{\theta}}\right)-\frac{\partial \hat{x}_{m}}{\partial \hat{\theta}}=\left(\frac{D^{2}}{\left(J P_{x}-D\right)\left((1-J) P_{x}+D\right)}\right) \frac{\partial \hat{x}_{m}}{\partial \hat{\theta}} \equiv L \frac{\partial \hat{x}_{m}}{\partial \hat{\theta}}
$$

where

$$
D=\int_{-\infty}^{\theta^{\prime}} \frac{\partial J\left(t, z^{\prime}\right)}{\partial t} \frac{\partial P\left(t \mid z^{\prime}, x^{\prime}\right)}{\partial x} \mathrm{~d} t
$$

Lemma 9 in the Technical Appendix shows that there always exists $\delta$ such that the lower bound of $L$ is increasing in $\beta$ and is bounded above 0 when $\beta$ approaches 1 . On the other hand, $L_{p s}$ is bounded from above with an upper bound $(1-\beta) / \beta$. Observe that $(1-\beta) / \beta$ is decreasing in $\beta$ and approaches 0 when $\beta$ approaches 1 . There must exist a threshold $\hat{\beta}$ such that, for any $\beta>\hat{\beta}$, there exists $\delta$ so that $L>L_{p s}$. By continuity, for any $\beta>\hat{\beta}$, there exists an interval $\left[\delta_{1}, \delta_{2}\right]$ so that for any $\delta \in\left[\delta_{1}, \delta_{2}\right], L>L_{p s}$, which implies that inequality (19) holds. Using the same logic as in Proposition 4, we obtain $\mathrm{d} \theta^{*}\left(z^{\prime}\right) / \mathrm{d} z<\mathrm{d} \theta_{p s}^{*}\left(z^{\prime}\right) / \mathrm{d} z<0$. The proposition follows.

Proof of Proposition 6. Consider a symmetric communication rule $S=[z-\Delta, z+$ $\Delta$ ]. It is straightforward to see that both $w(z, \cdot)$ and $q(S ; z, \cdot)$ are symmetric about $z$. Therefore, if $x_{i}=\underline{x}<z$ solves (20) with equality, then $x_{i}=\bar{x}=z+(z-\underline{x})>z$ also does.

To establish the existence of interval decision rules, we denote $\tilde{q}(\Delta)=q(S ; z, z-\Delta)$ and $\tilde{w}(\Delta)=w(z, z-\Delta)$. We need to establish that there exists $\Delta>0$, such that

$$
\tilde{q}(\Delta)=\frac{d_{1}+c_{1}-\tilde{w}(\Delta)\left(c_{0}+c_{1}\right)}{d_{0}+d_{1}}
$$

Note that $\tilde{w}(0)=w(z, z)$ and $\lim _{\Delta \rightarrow \infty} \tilde{w}(\Delta)=0$, while $\lim _{\Delta \rightarrow 0} \tilde{q}(\Delta)=0$ and $\lim _{\Delta \rightarrow \infty} \tilde{q}(\Delta)=$ 1. Therefore, given the condition stated in the proposition, there exists $\Delta^{*}>0 \mathrm{such}$ that the equation holds. Moreover, given $S^{*}=\left[z-\Delta^{*}, z+\Delta^{*}\right], q\left(S^{*} ; z, x_{i}\right)$ is increasing then decreasing in $x_{i}$, with a peak at $x_{i}=z$. Thus,

$$
q\left(S^{*} ; z, x_{i}\right) \geq q\left(S^{*} ; z, \underline{x}\right)=\frac{d_{1}+c_{1}-w(z, \underline{x})\left(c_{0}+c_{1}\right)}{d_{0}+d_{1}} \geq \frac{d_{1}+c_{1}-w\left(z, x_{i}\right)\left(c_{0}+c_{1}\right)}{d_{0}+d_{1}}
$$

if and only if $x_{i} \in S^{*}$. 


\section{References}

Acemoglu, D., A. Ozdaglar, and A. ParandehGheibi (2010). Spread of (mis)information in social networks. Games and Economic Behavior 70(2), 194-227.

Allport, G.W. and L.J. Postman (1947). The Psychology of Rumor. New York,: H. Holt and Company.

Angeletos, G.M. and I. Werning (2006). Crises and crices: information aggregation, multiplicity, and volatility. American Economic Review 96(5), 1720-1736.

Banerjee, A.V. (1993). The economics of rumours. Review of Economic Studies 60(2), 309-327.

Bernhardt, D. and M. Shadmehr (2011). Collective action with uncertain payoffs: Coordination, public signals and punishment dilemmas. American Political Science Review 105(4), 829-851.

Bilefsky, D. (2009). Celebrating revolution with roots in a rumor. The New York Times, November 18, p. 16.

Bommel, J.V. (2003). Rumors. Journal of Finance 58(4), 1499-1519.

Buchner, H.T. (1965). A theory of rumor transmission. Public Opinion Quarterly 29(1), 54-70.

Bueno de Mesquita, E. (2010). Regime change and revolutionary entrepreneurs. American Political Science Review 104(3), 446-466.

Edmond, C. (2011). Information manipulation, coordination, and regime change. Working Paper 17395, National Bureau of Economic Research.

Elias, N. and J.L. Scotson (1994). The Established and The Outsiders: A Sociology Enquiry into Community Problems, 2d ed. SAGE Publications.

Esfandiari, G. (2010). The Twitter devolution. Foreign Policy, June 7.

Gambetta, D. (1994). Godfather's gossip. European Journal of Sociology 35(2), 199-223.

Gentzkow, M. and J.M. Shapiro (2006). Media bias and reputation. Journal of Political Economy 114(2), 280-316.

Goldstone, J.A. (1994). Revolutions: Theoretical, Comparative, and Historical Studies, 2d ed. Fort Worth: Harcourt Brace.

Knapp, R.H. (1944). A psychology of rumor. The Public Opinion Quarterly 8(1), 22-37.

Milgrom, P.R. (1981). Good news and bad news: Representation theorems and applications. Bell Journal of Economics 12(2), 380-391. 
Morris, S. and H.S. Shin (1998). Unique equilibrium in a model of self-fulfilling currency attacks. American Economic Review 88(3), 587-597.

Morris, S. and H.S. Shin (2003). Global games: Theory and applications. In M. Dewatripont, L.P. Hansen, and S.J. Turnovsky, eds., Advances in Economics and Econometrics: Theory and Applications, Eighth World Congress, Vol. 1. Cambridge: Cambridge University Press.

Nkpa, N.K.U. (1977). Rumors of mass poisoning in Biafra. Public Opinion Quarterly 41(3), 332-346.

Peterson, W.A. and N.P. Gist (1951). Rumor and public opinion. American Journal of Sociology 57(2), 159-167.

Shadmehr, M. and D. Bernhardt (Forthcoming). State censorship. American Economic Journal: Microeconomics.

Skocpol, T. (1979). States and Social Revolutions: A Comparative Analysis of France, Russia, and China. New York: Cambridge University Press.

Suen, W. (2010). Mutual admiration clubs. Economic Inquiry 48(1), 123-132.

Turner, M.E., A.R. Pratkanis, P. Probasco, and C. Leve (1992). Threat, cohesion, and group effectiveness: Testing a social identity maintenance perspective on group think. Journal of Personality and Social Psychology 63(5), 781-796.

Zhang, N. (2009). Rumors and mobilization in China's contentions politics. Ph.D. dissertation, Chinese University of Hong Kong. 